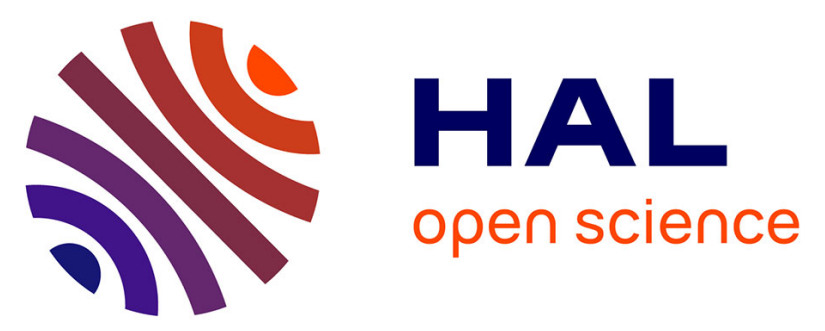

\title{
Mars H Escape Rates Derived from MAVEN/IUVS Lyman Alpha Brightness Measurements and their Dependence on Model Assumptions
}

Michael S. Chaffin, Jean-Yves Chaufray, Justin Deighan, M. M. Schneider, M. Mayyasi, J. T. Clarke, E. Thiemann, S. K. Jain, M. M. J. Crismani, Arnaud Stiepen, et al.

\section{To cite this version:}

Michael S. Chaffin, Jean-Yves Chaufray, Justin Deighan, M. M. Schneider, M. Mayyasi, et al.. Mars H Escape Rates Derived from MAVEN/IUVS Lyman Alpha Brightness Measurements and their Dependence on Model Assumptions. Journal of Geophysical Research. Planets, 2018, 123 (8), pp.2192-2210. 10.1029/2018JE005574 . insu-01835118

\section{HAL Id: insu-01835118 https://hal-insu.archives-ouvertes.fr/insu-01835118}

Submitted on 13 Jan 2021

HAL is a multi-disciplinary open access archive for the deposit and dissemination of scientific research documents, whether they are published or not. The documents may come from teaching and research institutions in France or abroad, or from public or private research centers.
L'archive ouverte pluridisciplinaire HAL, est destinée au dépôt et à la diffusion de documents scientifiques de niveau recherche, publiés ou non, émanant des établissements d'enseignement et de recherche français ou étrangers, des laboratoires publics ou privés. 


\section{Journal of Geophysical Research: Planets}

\section{RESEARCH ARTICLE}

10.1029/2018JE005574

\section{Special Section:}

Mars Aeronomy

Key Points:

- Mars $\mathrm{H}$ loss rates are inferred from MAVEN IUVS Lyman alpha profiles

- New fitting techniques produce near-perfect matches to data using either hot hydrogen or deuterium - New fitting techniques produce near-perfect matches to data using either hot hydrogen or deuterium

Correspondence to:

M. S. Chaffin,

michael.chaffin@colorado.edu

Citation:

Chaffin, M. S., Chaufray, J. Y.,

Deighan, D., Schneider, N. M.,

Mayyasi, M., Clarke, J. T., et al. (2018).

Mars $\mathrm{H}$ escape rates derived from

MAVEN/IUVS Lyman alpha brightness measurements and their dependence on model assumptions. Journal Geophysical Research

Planets, 123, 2192-2210.

https://doi.org/10.1029/2018JE005574

Received 16 FEB 2018

Accepted 26 JUN 2018

Accepted article online 5 JUL 2018

Published online 30 AUG 2018
○2018. American Geophysical Union. All Rights Reserved.

\section{Mars H Escape Rates Derived From MAVEN/IUVS Lyman Alpha Brightness Measurements and Their Dependence on Model Assumptions}

\author{
M. S. Chaffin ${ }^{1}$ (D) , J. Y. Chaufray ${ }^{\text {DD , J. Deighan }}{ }^{1}$ D, N. M. Schneider ${ }^{1}$, M. Mayyasi ${ }^{3}$, , \\ J. T. Clarke $^{3}$, E. Thiemann ${ }^{1}$ D , S. K. Jain ${ }^{1}$ D, M. M. J. Crismani ${ }^{1}$ (D) , A. Stiepen ${ }^{4}$, , \\ F. G. Eparvier' ${ }^{1}$ D, W. E. McClintock' ${ }^{1}$, A. I. F. Stewart ${ }^{1}$, G. M. Holsclaw ${ }^{1}$ (D), \\ F. Montmessin' ${ }^{\text {iD }}$, and B. M. Jakosky ${ }^{1}$ (D) \\ ${ }^{1}$ Laboratory for Atmospheric and Space Physics, University of Colorado Boulder, Boulder, CO, USA, ${ }^{2}$ LATMOS/IPSL, \\ Guyancourt, France, ${ }^{3}$ Center for Space Physics, Boston University, Boston, MA, USA, ${ }^{4}$ Laboratoire de Physique \\ Atmosphérique et PlanÃl'taire, Space sciences, Technologies and Astrophysics Research Institute, University of Liége, \\ Liége, Belgium
}

Abstract Mars has lost a large fraction of its water to space, with the $\mathrm{H}$ component of this loss thought to occur mainly as a result of thermal (Jeans) escape from the upper atmosphere. Constraints on $\mathrm{H}$ loss have historically been made using hydrogen Lyman alpha $(121.6 \mathrm{~nm})$ light scattered in the planet's extended upper atmosphere or corona. Here we employ observations from the Mars Atmosphere and Volatile Evolution (MAVEN) mission's Imaging Ultraviolet Spectrograph (IUVS) to constrain H escape in December 2014 and August 2016, when MAVEN observed the dayside corona at low latitude. To obtain adequate fits and address systematic sources of uncertainty including instrument calibration, we fit in exobase number density and escape rate instead of density and temperature, employing Markov Chain Monte Carlo techniques. This produces better model fits to data than most previous analyses. When we assume a single population of $\mathrm{H}$ atoms, we obtain $\mathrm{H}$ temperatures inconsistent with expected trends and a shape mismatch between observed and modeled profiles, similar to previous studies. Introducing either a second population of $\mathrm{H}$ (at a distinct temperature and density) or adding deuterium to the corona allows for essentially perfect fits. Despite this model ambiguity, derived loss rates for both periods are within a factor of four, 3.3-8.8 $\times 10^{8} \mathrm{~cm}^{-2} / \mathrm{s}$ in December $2014\left(L_{s} \sim 250\right)$ and $0.6-2.3 \times 10^{8} \mathrm{~cm}^{-2} / \mathrm{s}$ in August $2016\left(L_{s} \sim\right.$ 200). These rates are similar to those found in prior studies and confirm the known seasonal trend — doing so while incorporating the substantial uncertainty in absolute calibration insufficiently explored by previous studies.

\section{History of H Escape Measurements}

The Martian atmosphere is mostly $\mathrm{CO}_{2}$, which is stabilized against photodissociation by an atmospheric odd-hydrogen cycle in which water vapor dissociation products catalyze $\mathrm{CO}_{2}$ recombination (McElroy, 1972 Parkinson \& Hunten, 1972). Not all of the $\mathrm{H}$ involved in this cycle recombines into water; some forms molecular hydrogen, which does not condense as it carries $\mathrm{H}$ from the lower to the upper atmosphere. Once there, $\mathrm{H}_{2}$ is broken by ionospheric chemistry, and $\mathrm{H}$ diffuses to the upper atmosphere, where collisions are infrequent and atoms travel on ballistic trajectories and can escape the planet's atmosphere. Measurements of the collisionless corona can be used to estimate the loss of $\mathrm{H}$ to space today and infer the total loss throughout time. This loss has been significant: at the present-day rate, at least $10 \%$ of the $\mathrm{H}$ initially present as water on Mars would have escaped to space (Chaffin et al., 2014). D/H isotope fractionation measured in near-surface water vapor suggests the integrated loss is much higher, with more than 80\% loss (Krasnopolsky, 2015; Villanueva et al., 2015). However, accurate interpretation of these measurements depends on precise knowledge of the Martian water inventory and the exchange rates between different storage reservoirs (crustal ice, polar caps, etc.), including atmospheric water vapor. Measurement of the contemporary loss rate offers another path to determining water loss.

The most common means of constraining present-day $\mathrm{H}$ loss is ultraviolet remote sensing, which observes radiative transitions of $\mathrm{H}$ atoms in the thermosphere and corona. Lyman series measurements probe the 
ground state $\mathrm{H}$ column, capturing the vast majority of planetary $\mathrm{H}$ atoms. Instrument design issues including a lack of transmissive materials at extreme ultraviolet wavelengths make Lyman alpha much easier to measure than other lines in the Lyman series. This advantage is amplified by the relative strength of the line and the correspondingly higher brightness of the Sun at this wavelength. As a result, most constraints on Mars $\mathrm{H}$ escape have been made using $121.6 \mathrm{~nm}$ Lyman alpha light. Multiple spacecraft have measured this line and inferred $\mathrm{H}$ escape rates at Mars, including Mariner 6 and 7 (Anderson \& Hord, 1971), Mars 2, 3, and 5 (Babichenko et al., 1977; Dostovalov \& Chuvakhin, 1973), Mariner 9 (Anderson, 1974), Mars Express (Chaufray et al., 2008; Chaffin et al., 2014), and the Hubble Space Telescope (Bhattacharyya et al., 2015; Clarke et al., 2014). Rosetta (Feldman et al., 2011) measured Lyman alpha but based on retrievals of $\mathrm{H}$ escape on the simultaneously measured Lyman beta (102.6 nm) line. Most recently, Mars Atmosphere and Volatile Evolution (MAVEN) observations of the thermosphere and corona have been made (Chaffin et al., 2015; Clarke et al., 2017; Mayyasi et al., 2017), but not yet quantitatively linked to escape rates, owing to unresolved issues with instrument calibration and growing recognition of model incompleteness, discussed more fully below.

Other techniques have been used to infer $\mathrm{H}$ loss rates and variability at Mars. These include the occurrence and variability of proton ion cyclotron waves (Bertucci et al., 2013; Romanelli et al., 2016), hydrogen pickup ions (Dubinin et al., 2006; Rahmati et al., 2017; Yamauchi et al., 2015), energetic neutral atoms resulting from solar wind proton-coronal hydrogen charge exchange (Futaana et al., 2006; Gunell et al., 2006), and energetic ionospheric protons resulting from the impact of these neutrals with the collisional atmosphere at lower altitudes (Halekas, 2017; Halekas et al., 2015; Halekas et al., 2017). Because the processes producing these signatures are less direct, these observables have been less frequently used to place quantitative constraints on $\mathrm{H}$ loss.

Taken together, these studies suggest a large seasonal variation in $\mathrm{H}$ escape, with low rates near $10^{7} \mathrm{~cm}^{-2} / \mathrm{s}$ near northern summer solstice and aphelion, and rates a factor of 10-100 higher near southern summer solstice and perihelion. The mechanism for the variation is seasonal, but the relative contribution of the underlying processes is unknown, with some authors inferring the presence of a nonthermal $\mathrm{H}$ component presumably produced by ionospheric chemistry or charge exchange driven by higher extreme ultraviolet insolation at perihelion (Bhattacharyya et al., 2015; Chaffin et al., 2014; Chaufray et al., 2008; Clarke et al., 2014). However, the recent measurements of Halekas et al. (2017) show escape rates peaking at southern summer solstice rather than perihelion, suggesting that direct energization of upper atmospheric hydrogen is not the source of the enhancement. Instead, lower-to-middle atmosphere transport of water is a more likely culprit for elevated escape. Modeling studies suggest that this hypothesis can explain the observed variation (Chaffin et al., 2017), and measurements of enhanced water at middle atmospheric altitudes suggest the seasonal presence of water (Fedorova et al., 2018; Heavens et al., 2018; Maltagliati et al., 2013), but a definitive signature of water transport enhanced escape has yet to be detected. Future studies of correlated time variability in middle atmospheric $\mathrm{H}_{2} \mathrm{O}$ and $\mathrm{H}$ and/or detection of intermediate chemical species would secure support for this enhancement mechanism.

\section{Description of Observations and Data Set}

MAVEN remote sensing is performed with the Imaging Ultraviolet Spectrograph (IUVS), a far- to mid-UV instrument described in detail by McClintock et al. (2015). The instrument observes simultaneously in two channels that cover the far-UV (110-190 $\mathrm{nm})$ at 0.6-nm resolution and the mid-UV (180-340 $\mathrm{nm})$ at 1.2-nm resolution. Integration times and observing modes are extremely flexible, because the spectrograph contains an internal scan mirror and is mounted on an articulated payload platform with two gimbal steering. To be concise, we describe here only the characteristics of IUVS relevant to the observations analyzed. The instrument is sensitive to $\mathrm{H}$ Lyman alpha, which is ubiquitous in IUVS spectra because Lyman alpha sunlight is scattered by hydrogen in the interplanetary and interstellar medium, and in the Mars atmosphere. At Mars, Lyman alpha photons are multiply scattered, with typical vertical optical depth at the exobase of order 1-10. This multiple scattering means that Lyman alpha observations must be interpreted with a radiative transfer model to place quantitative constraints on $\mathrm{H}$ densities and escape rates. Observations useful for this purpose are made by IUVS on the side segments of MAVEN's elliptical orbit, when the instrument is pointed inertially across the orbit parallel to the semiminor axis, with the slit parallel to the limb. In this geometry, IUVS builds an altitude profile of coronal emission using spacecraft motion. Because the spacecraft orbit was designed to sample a wide range of solar latitude and longitude at periapsis over the length of the mission, the precession of the orbit is fast, so that periapsis passes from day to night on approximately seasonal timescales 


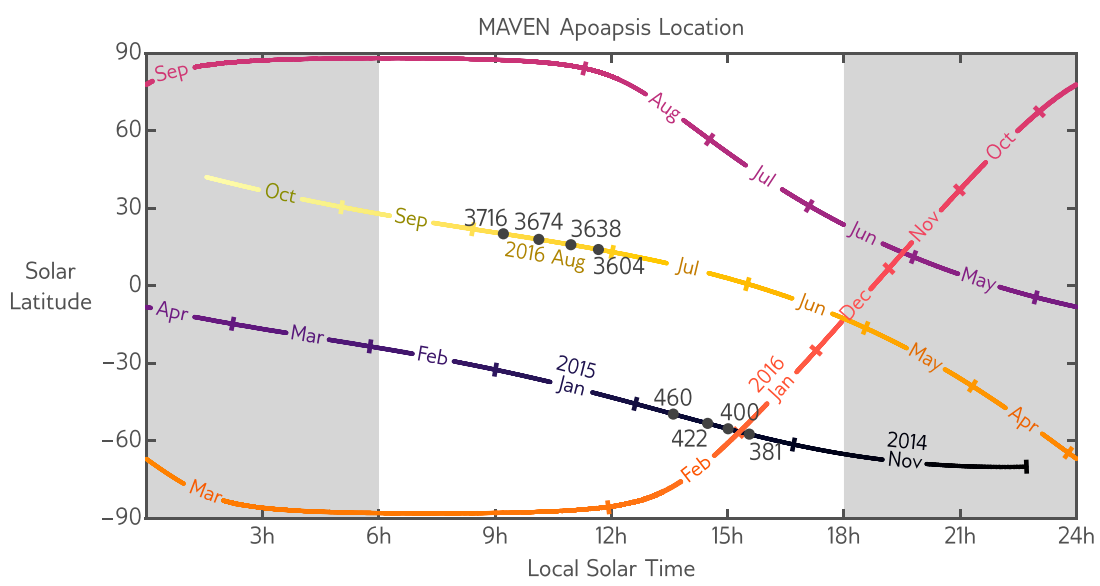

Figure 1. MAVEN apoapsis location as a function of time. Apoapsis coordinates are given in the solar-centered reference frame, with the nightside of the planet indicated with gray shading. Over the course of 2 years, the MAVEN orbit precesses significantly around the planet, with apoapsis crossing the dayside near the solar equator only twice, in late 2014/early 2015 and in middle to late 2016. The location of the eight orbits studied in this work are indicated by orbit number. MAVEN = Mars Atmosphere and Volatile Evolution.

(Jakosky et al., 2015). In addition, during the prime mission the orbit periapsis swept across the dayside, leaving the orbit sides and apoapsis on the nightside of the planet.

For this study, we use dayside observations of the corona at low latitude, excluding terminator, nightside, and high-latitude data. The excluded data, gathered far from the subsolar point, are not ideal for placing constraints on $\mathrm{H}$ escape: their interpretation requires a detailed understanding of the scattering of Lyman alpha around the limb by dayside hydrogen at a different density and temperature, requiring modeling that incorporates variations with local time and latitude. In addition, the intensity of observed $\mathrm{H}$ Lyman alpha is dimmer at higher solar zenith angles. Dayside coronal scans are preferable, but the time span over which they are available is limited.

Over the course of the MAVEN mission so far, two time periods contain dayside coronal scans at relatively low solar latitude. Figure 1 shows the evolution of the orbit apoapsis and indicates the time periods of interest. In December of $2014\left(L_{s} 250\right)$, observations were made across southern solar latitudes, sweeping from observations looking nightward across the evening terminator to cross-noon observations made from the dawnside of the planet. In August of $2016\left(L_{s} 200\right)$, apoapsis was again on the dayside, with cross-noon observations made from northern latitudes at dawn toward southern latitudes at dusk at the beginning of the period, precessing to south pointing morning observations at the end. During both of these periods, IUVS alternated pointing control with the STATIC instrument (McFadden et al., 2015), observing on the side segment of one out of every two orbits. IUVS alternates observing modes between standard and high-resolution on the side segments of the orbit; as a result we obtain high-altitude coronal scans on every fourth orbit.

Because the analysis we perform is computationally intensive, we selected a representative subset of the available orbits to study. For each time period we selected four orbits, spanning the months of December 2014 and August 2016 and representing about $11 \%$ of the available data set. Figure A1 shows all of the coronal profiles from these time periods, demonstrating that the variation observed is smooth. In December 2014, every eighth observation was selected, beginning with orbit 381. This set captures orbit 422, which was found to be relatively noise free in oxygen corona analysis performed by Deighan et al. (2015). In August 2016, every eighth observation was selected beginning with orbit 3604 . The month-long time span captured in these two periods should be sufficient to reveal some short-term variability, and the difference in Mars season between the two periods should enable some analysis of seasonal variability, keeping in mind that the two periods occur in different Mars years. Because the changes observed in coronal profiles are smooth across both time periods, and because the profiles we select have approximately the same signal to noise as the others (see, for example, the range of profiles presented in Chaffin et al. (2015)), we do not expect that our selection of profiles to fit will bias our analysis. Simultaneously fitting more profiles is possible but expensive: the analysis presented here required $24 \mathrm{hr}$ on 48 cores and $200 \mathrm{~GB}$ of RAM. 

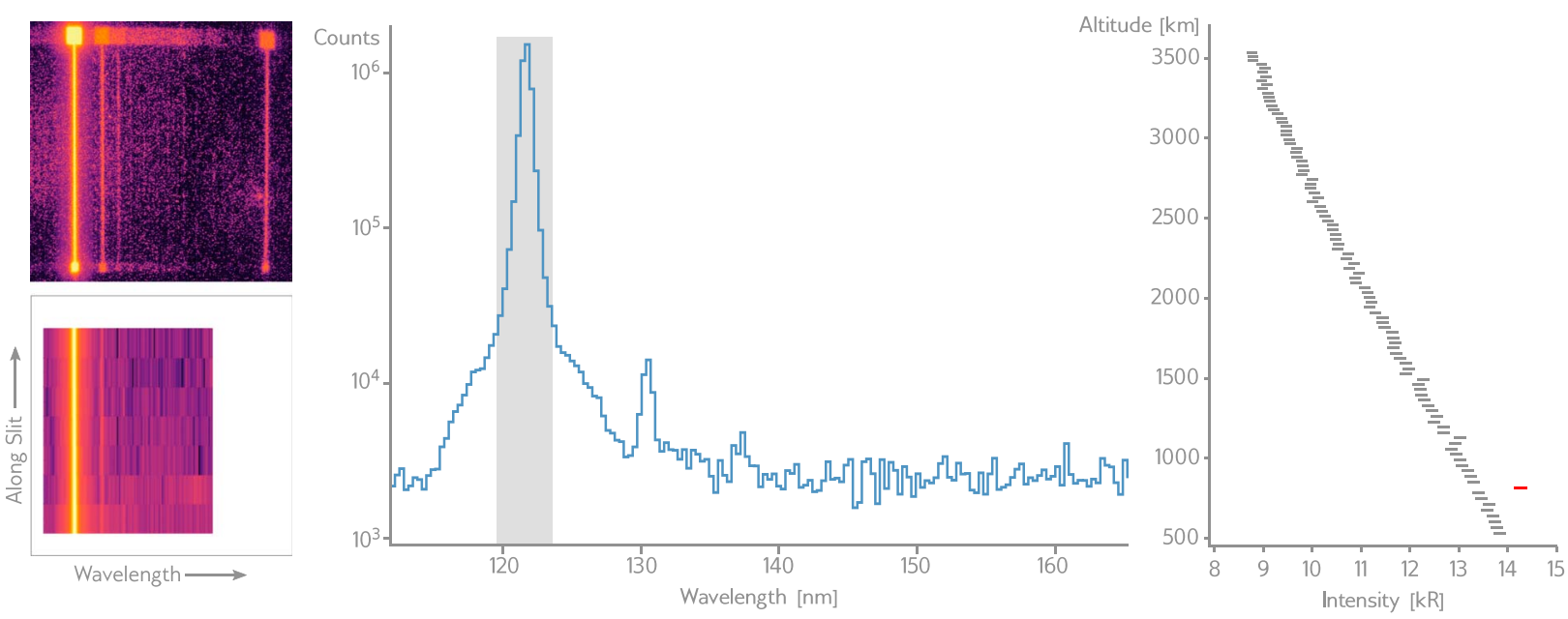

Figure 2. Summary of data reduction. (upper left) The native format of IUVS is a $1024 \times 1024$ spectral image. (lower left) In coronal scan mode, the data are binned on board the spacecraft to reduce data volume, producing seven spatial bins that sample along the slit and at least 128 spectral bins. (center) We average the spectrum along the slit and integrate over the Lyman alpha line (region of integration shaded) to produce a brightness profile. We ignore other coronal features, including coronal oxygen at $130.4 \mathrm{~nm}$. (right) We exclude outliers such as the red point from the resulting profiles using a procedure described in the text. This produces a profile to which models can be fit.

We show our data reduction procedure in Figure 2. The native format of the data is a $1024 \times 1024$ image of the spectrum, which is binned onboard the instrument to reduce the data volume for transmission to Earth. After binning, most coronal scan data share a common format with 128-256 spectral bins encompassing the full wavelength range of the instrument. Seven spatial bins sample the length of the narrow airglow slit and omit the slit keyholes, which are typically used to observe stellar occultations rather than airglow. In coronal scan mode, the spectrum is imaged and recorded on a 15-s cadence, resulting in about 150 observations spanning a tangent point altitude range of approximately 400 to $4,000 \mathrm{~km}$. For each observation, we average the spectra along the slit to produce a single spectrum at each altitude. To isolate the Lyman alpha line, we work in detector counts, summing the intensity across bins capturing the line core and subtracting the average value in fixed background bins selected on both sides. The line wings result from grating scatter inside the instrument and do not contribute greatly to the signal. We then calibrate the counts to Lyman alpha intensities using the sensitivity curve of the instrument, which can be obtained by taking the ratio of calibrated data to dark subtracted counts in the Planetary Data System hosted data products. Because calibration at Lyman alpha is uniquely difficult, the sensitivity of the instrument at this wavelength has been subjected to a high degree of scrutiny by Mayyasi et al. (2017). Our fitting technique, described below, includes a free parameter to adjust the instrument calibration within uncertainties to better fit the model. We discuss this issue further below and in Appendix B, concluding that all model fits prefer that the instrument calibration be adjusted to make IUVS intensities brighter by $10-20 \%$, at the edge of what is allowed by this earlier cross-calibration analysis. The combination of slit-averaging, integration, and calibration adjustment we apply results in a brightness value insensitive to flat field defects, since these can be removed by adjusting the applied absolute calibration factor. The random uncertainty at each altitude is obtained by propagation of reported random uncertainties from the individual detector bins, adding these in quadrature and dividing by the square root of the number of spatial bins to obtain the standard deviation of the mean. This propagation of errors assumes that all spectral bins are perfectly correlated and spatial bins are uncorrelated.

Once Lyman alpha altitude profiles have been constructed for each orbit, we filter the data to remove instrument artifacts. IUVS detects photoevents with an intensified an intensified imaging detector which is also to high-energy particles such as cosmic rays, which can introduce spurious high signal values. Similarly, particle events in dark frames can produce spurious low signals. To remove outliers in each profile, we perform linear fits to overlapping subsamples of each profile, using a rolling window of 30 points across the whole profile to produce the subsamples. We construct the linear fits from the middle $50 \%$ of data points in each subset, excluding the upper and lower quartile of the data. We then flag and remove points that deviate from the linear fit to any subset by more than 20 times the median absolute deviation of fit/data differences for that subsample. This procedure is somewhat arbitrary, but removes obvious outliers without affecting marginal 
cases, and retains the vast majority of the data. Typically, fewer than five points are removed from each profile in this way.

In addition to IUVS-measured coronal brightnesses, we require the solar brightness at Lyman alpha to retrieve the abundance of hydrogen in the corona. MAVEN carries a solar Extreme Ultraviolet Monitor (EUVM) designed in part to measure solar Lyman alpha with a filter diode (Eparvier et al., 2015). EUVM measures on a 1-s cadence, and the best measurement of the solar line strength is obtained near apoapsis when the Sun-MAVEN line of sight passes through the smallest column of Mars coronal H. Orbit-by-orbit line-integrated Lyman alpha brightnesses were produced from the EUVM data and used to interpret the coronal profiles. We interpolate these EUVM orbit-by-orbit EUVM brightnesses to the time of the coronal observations, using a linear interpolation where data are available on both sides of the orbit within 2 days. In cases where EUVM data are more than 2 days distant from the IUVS observation on either side, we perform no analysis. For all of the orbits selected for study here, we have EUVM data on the orbit in question and the prior orbit, but for orbits 422 and 3674 we lack data on the subsequent orbit and interpolate using orbits 424 and 3681, respectively, with what we expect to be a minimal effect on the derived solar brightness. Because we require line center solar brightnesses, we obtain these from the band-integrated value using the relation of Emerich et al. (2005). Both the EUVM data and the band-integrated to line-center relationship carry absolute uncertainties on the order of several percent, which can be accounted for with our previously introduced prior on the absolute calibration of IUVS.

\section{Retrieval Technique}

Because Lyman alpha photons are multiply scattered in the Mars corona, a radiative transfer model is required to retrieve escape rates from intensity measurements. We employ a forward modeling approach, coupling a parametric hydrogen density model to a photon scattering model. These models compute the brightness produced by a given model atmosphere, which can be compared with observations. Minimizing the chi-squared difference of the data and the model then yields the best fit atmospheric parameters. Historically, these best fit parameters have been obtained by exhaustive searches of parameter space or through the use of traditional minimization techniques such as Levenberg-Marquardt.

Optically thick scattering calculations are time intensive; to minimize the number of calculations we must perform, we make simplifying assumptions in both the density and radiative transfer modeling. For the most part, these assumptions do not differ from those previously adopted in $\mathrm{H}$ coronal retrievals at Mars (Bhattacharyya et al., 2015; Chaffin et al., 2014; Chaufray et al., 2008; Clarke et al., 2014) and allow us to obtain adequate fits to observed coronal profiles. Discussion of how these assumptions could be relaxed in future work is provided in sections 4 and 5 .

The density model is identical to that presented in Chaufray et al. (2008). The model is spherically symmetric and accepts two parameters: the number density and temperature of hydrogen at the Mars exobase, assumed to be at $200 \mathrm{~km}$. The $\mathrm{CO}_{2}$ density at $80 \mathrm{~km}$ is fixed at $2.67 \times 10^{13} \mathrm{~cm}^{-3}$ for all fitted profiles, and its temperature is assumed identical to the $\mathrm{H}$ temperature. Temperatures below the exobase are modeled using an analytic profile from Krasnopolsky (2002), which interpolates from the model exobase temperature to $125 \mathrm{~K}$ at $90 \mathrm{~km}$. The exosphere is assumed isothermal at the exobase temperature. All velocity distributions are assumed to be Maxwellian at the temperature corresponding to the altitude of interest. The $\mathrm{CO}_{2}$ is assumed to have a hydrostatic density profile determined from the temperature-dependent scale height. The $\mathrm{H}$ density profile is determined above the exobase assuming ballistic orbits, resulting in a Chamberlain exosphere (Chamberlain, 1963). Below the exobase, $\mathrm{H}$ densities are determined by solving the diffusion equation with the exobase density as a boundary condition. Below $120 \mathrm{~km}$, the $\mathrm{H}$ density is assumed constant. This last assumption does not strongly affect our results because the atmosphere is mostly opaque at Lyman alpha at these altitudes due to $\mathrm{CO}_{2}$ absorption (Chaufray et al., 2008).

Our radiative transfer model is descended from Anderson and Hord (1977). We assume Gaussian line shapes and complete frequency redistribution to solve the radiative transfer equation. To save time when fitting data from many spacecraft orbits, we precompute the atmospheric scattering on a grid of hydrogen density and temperature, resulting in source functions that are integrated along observation lines of sight for comparison with spacecraft measurements. This model library populates a grid in $\mathrm{H}$ density-temperature space, running from $100 \mathrm{~K}$ to $1600 \mathrm{~K}$ in increments of $10 \mathrm{~K}$ and from $10^{5} \mathrm{~cm}^{-3}$ to $10^{7} \mathrm{~cm}^{-3}$ in 100 logarithmic steps. 
The major addition of this work to the retrieval algorithm is the estimation of model parameters using probabilistic Markov Chain Monte Carlo (MCMC) as implemented by the python emcee package (Foreman et al., 2013). In MCMC, an N-dimensional function is explored by a large number of walkers, each of which occupies a position in the $\mathrm{N}$-dimensional parameter space. We use the python emcee package (Foreman et al., 2013) to perform MCMC retrievals. At each time step, the walker positions are updated randomly so that their density distribution approaches proportionality with the sampled function. In our work, these walkers explore the log posterior probability function, representing the sum of the log likelihood of the model fit (chi-squared times -1 ) and the log prior probability of the parameters, representing any expectations about the parameters resulting from previous analysis or physical limits. After enough steps have been taken, the walkers cluster near the maximum of this function, and their coordinates are distributed according to the posterior probability of the modeled parameters, producing the best fit parameters and a measure of their covariance. We determine that enough steps have been taken by visual inspection of the chains and the model profiles to determine that (1) all chains oscillate around the same best fit values, and (2) the fits are adequate. In future work, we will apply more stringent tests of convergence, including application of the Gelman-Rubin statistic (Brooks \& Gelman, 1998; Gelman \& Rubin, 1992). For now, the excellent agreement of model and data gives us confidence that the fit values are appropriate.

MCMC has several advantages over traditional minimization techniques. First, because it directly samples the likelihood function, MCMC allows for easy propagation of uncertainties and marginalization of nuisance parameters: computing the desired quantity for all walkers immediately produces a sampled distribution of parameter values. Second, MCMC allows for straightforward inclusion of systematic uncertainties shared across observations, making it easy for us to propagate the uncertain instrument calibration at Lyman alpha to our derived escape rates. Because of the strong degeneracy between parameters encountered in fitting this optically thick emission, and the 10-20\$ uncertainty in absolute calibration of all spaceborne instruments at Lyman alpha, these features of MCMC are required for a rigorous treatment of uncertainty in derived escape rates. Although many of these issues have been discussed before (Bhattacharyya et al., 2017), we are to our knowledge the first to treat this problem with the required level of statistical rigor.

To better understand the systematic uncertainties that may result from different assumed models, we perform three independent fits to the brightness profile assuming that the exobase is populated by Maxwellian thermal $\mathrm{H}$ only, by a combination of two Maxwellian populations at different densities and temperatures, and a thermal $\mathrm{H}$ and $\mathrm{D}$ population at the same temperature but different densities.

First, we assume a thermal spherically symmetric Maxwellian population with a single density and Jeans escape flux (determined by exobase temperature). We tabulate intensity profiles in this parameter space on a grid in density $\left(5 \times 10^{4}-1 \times 10^{7} \mathrm{~cm}^{-3}\right.$ in 100 logarithmic steps) and escape flux $\left(5 \times 10^{6}-5 \times 10^{9} \mathrm{~cm}^{-2} / \mathrm{s}\right.$ in 100 logarithmic steps), interpolating the grid profiles and fitting the interpolated intensities to the data. Because the physical-radiative transfer model inputs are density and temperature, we convert each density-escape flux pair into a density-temperature pair for model input, using the Jeans escape relationship

$$
\phi=n \frac{v_{\mathrm{mp}}}{2 \pi^{1 / 2}}(1+\lambda) e^{-\lambda}, \quad v_{\mathrm{mp}}=\sqrt{\frac{2 k T}{m}}, \quad \lambda=\frac{G M m}{k T r_{\mathrm{exo}}},
$$

where $\phi$ is the exobase escape flux, $n$ is the exobase density, $T$ is the exobase temperature, $v_{\mathrm{mp}}$ is the most probable Maxwell-Boltzmann velocity, $\lambda$ is the escape or Jeans parameter, $k$ is the Boltzmann constant, $r_{\text {exo }}$ is the exobase radius, $m$ is the mass of the hydrogen atom, $M$ is the planetary mass, and $G$ is the universal gravitational constant. There are two reasons we fit in density-escape flux space, even though the model is formulated in density-temperature space: first, this parameterization produces escape flux probability distributions without additional calculations; and second, parameterization in number density-temperature space produces an interaction between the native degeneracy of the space and the grid sampling that is likely to result in artificially high temperatures and low densities, as explained in Appendix C. The linear degeneracy present in the density-escape flux is effectively explored by the emcee MCMC routine we employ because this routine is affine invariant and therefore insensitive to linear transformations of the fitted parameters.

Second, we assume two components at the exobase: a thermal population, computed exactly as in the purely thermal case, and a second population with an independent density $\left(100-5 \times 10^{4} \mathrm{~cm}^{-3}\right.$ in 50 logarithmic steps) and temperature (100-1600 K in $25 \mathrm{~K}$ increments). At the densities and temperatures used for this 
component, most combinations result in optically thin emission profiles. Because the temperature is unconstrained, this component is free to represent either a hot or a cold $\mathrm{H}$ component, depending on what best fits the profile. To obtain the total brightness profile, we sum the intensity profile resulting from each population, assuming no radiative interaction between the species. This assumption likely underestimates the density or temperature of the second component, because it neglects any overlap in velocity space that would allow the two populations to exchange photons. A full treatment of this photon exchange would require significant modification of core routines in our radiative transfer code, beyond the scope of the work we present here, and exceeding the fidelity of all previous treatments of hot $\mathrm{H}$ in the Martian corona. Nevertheless, our two-component fits present a less constrained exploration of the hot $\mathrm{H}$ population present at Mars than in previous studies (Bhattacharyya et al., 2015; Chaufray et al., 2008), because these studies fixed the temperature of one or both $\mathrm{H}$ components using a priori assumptions about the temperatures expected for these populations, without a simultaneous exploration of the parameter space. Our procedure therefore has the potential to more accurately reflect the conditions present in the atmosphere without assumptions and produce better fits to the observed profiles.

Third, we assume hydrogen and deuterium are both present at the exobase, with independent densities but a common temperature. Deuterium should be present at the exobase (Yung et al., 1989), but its potential impact on Lyman alpha observations has not been fully explored. The $\mathrm{D}$ and $\mathrm{H}$ Lyman alpha lines do not radiatively interact, because the line separation is $\sim 0.3$, while the thermal line width at $200 \mathrm{~K}$ is on the order of $0.01 \forall$. However, in the $\approx 1$-nm resolution observations we study here, $\mathrm{D}$ and $\mathrm{H}$ Lyman alpha cannot be resolved, potentially masking a D component. IUVS echelle observations, which observe D in the thermosphere (Clarke et al., 2017; Mayyasi et al., 2017), are too faint to resolve H and D in the corona. To extract deuterium abundances from these unresolved observations, we model the Lyman alpha signal as the sum of separately modeled $\mathrm{D}$ and $\mathrm{H}$ brightnesses. To simulate deuterium brightnesses, we produced new source functions and new tabulated intensities, which we computed using our $\mathrm{H}$ corona model with the mass of the $\mathrm{H}$ atom doubled. This produces an $\mathrm{H}$ component with a deuterium-like scale height, reflecting the altitude distribution of deuterium expected in the atmosphere and corona at the thermal $\mathrm{H}$ temperature. We use the same solar flux to simulate the two components, resulting in a slight underestimate of the D brightness, because the solar line brightness is slightly higher at the $\mathrm{D}$ line center than at the $\mathrm{H}$ line center, owing to the self-reversed solar line profile (Emerich et al., 2005).

For each observation, the model brightness is computed using the observed Lyman alpha brightness of the Sun as measured by EUVM(Eparvier et al., 2015). We combine the model information and the observations to produce the likelihood function:

$$
\mathcal{L}(\text { data } \mid \theta, \text { back, cal })=\sum\left(\frac{\text { cal } \cdot(\text { model }(\theta)+\text { back })-\text { data }}{\text { uncertainties }}\right)^{2}
$$

where the sum is taken over all altitudes and every profile studied. For each of the three assumptions (thermal, two component, and deuterium), this likelihood is a function of independent model parameters $\theta$ for each profile (representing densities and temperatures of the model components), as well as the assumed model scale factor cal, shared across all profiles and all altitudes, and the interplanetary hydrogen (IPH) background back, similarly constant and assumed to have two distinct values, one shared across all December 2014 observations and one common to the August 2016 observations. Including cal and back allows us to propagate systematic uncertainties in these parameters into derived model parameters and allows the model the freedom to fit the shape of the profile with some independence from the absolute intensities observed. The total number of parameters required to compute the likelihood function is 19 in the case of thermal fits, representing a density and escape flux for each of the eight profiles along with two background IPH intensities and a single shared calibration factor. For two-component fits, a thermal and nonthermal density and temperature requires four parameters per profile and results in 35 total parameters. The $D$ fits have $H$ and D densities at a shared temperature for each profile, resulting in 27 parameters.

The posterior probability distribution of the parameters is proportional to the product of the likelihood distribution and the parameter priors, which we impose based on the expected behavior of each parameter. For each density, escape flux, and temperature fitted to the profiles, we assume a uniform prior throughout the range of allowed parameters. For the calibration, we assume a Gaussian prior in the range $0.5-1.5$, with a Gaussian distribution centered at 1.0 and a 1-sigma uncertainty of 0.3 , corresponding to the $30 \%$ absolute 

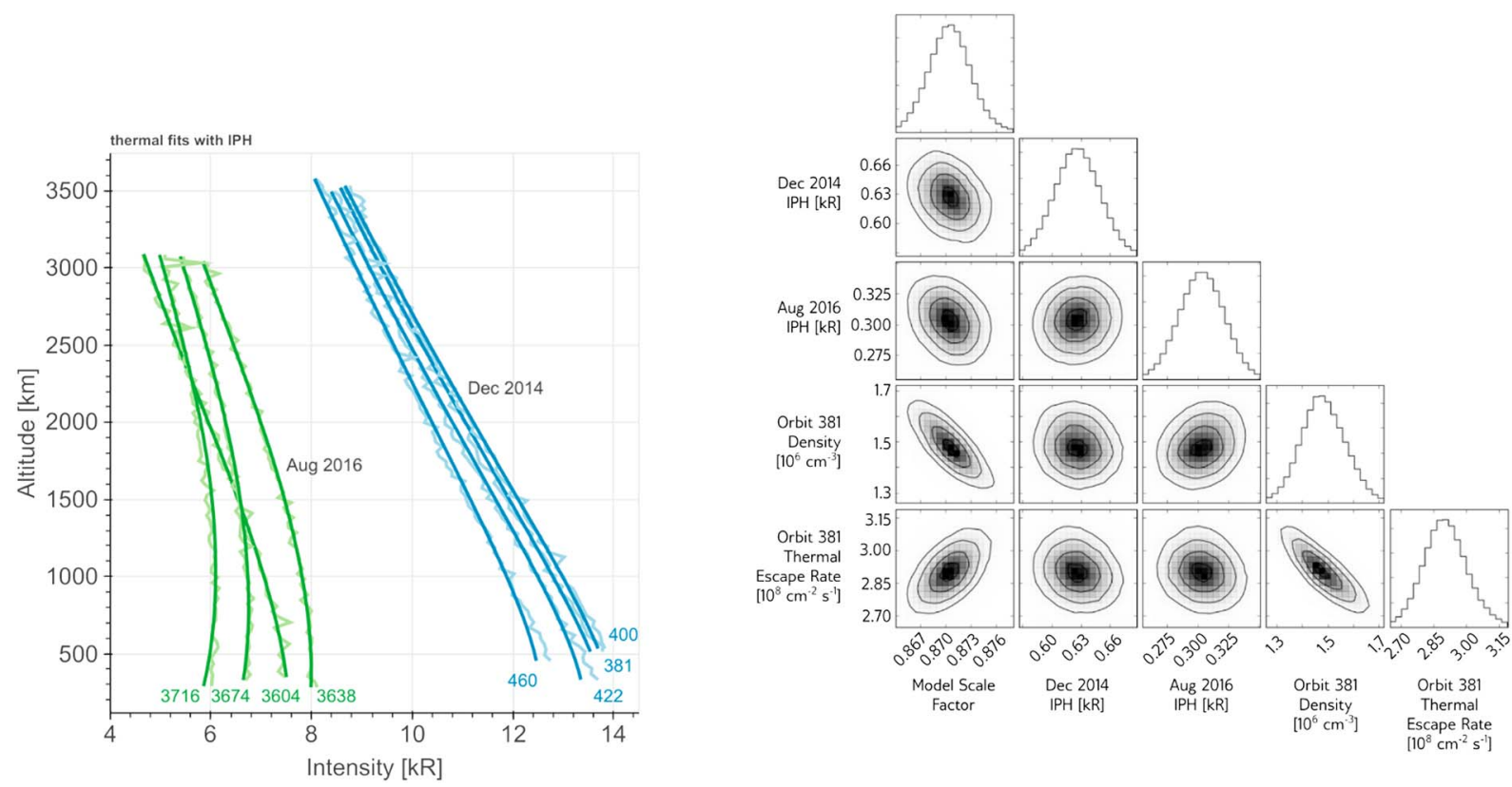

Figure 3. Thermal model best fits. (left) Observed brightness (lighter jagged lines) and best fit thermal models (dark smooth curves). Random uncertainties on data brightness are suppressed for clarity and can be inferred from data scatter or by reference to Figure 2. (right) Posterior distribution of fitted parameters for orbit 381, as an example of the general covariance of the fitted parameters. In this figure, the $\mathrm{N}$-dimensional probability distribution is visualized via its 2-D marginal distributions in every pair of variables and by the 1-D marginal distributions of each fitted variable. Contours are at 0.5 sigma intervals from $0.5-$ to 2-sigma. While model profiles match the mean intensity of the observations well, small differences in curvature and departures at low altitude suggest the need for an additional population of $\mathrm{H}$ or $\mathrm{D}$.

calibration uncertainty of IUVS in the far ultraviolet channel. The priors for the background IPH brightness were determined by extracting measured interplanetary brightnesses from the SWAN (solar wind anisotropies) instrument (Bertaux et al., 1995) and from an IPH model (Lallement et al., 1985), which gives values near $400 \mathrm{R}$ from SWAN and $600 \mathrm{R}$ from the model for December 2014 observations, and values near $300 \mathrm{R}$ and $400 \mathrm{R}$ for August 2016 observations. Given these values, we impose a Gaussian prior with $10 \%$ uncertainty of $500 \mathrm{R}$ for the December 2014 observations and 400 R for the August 2016 observations.

\section{Model Fits and Discussion of Inferred H Parameters}

Best fits assuming a single spherically symmetric Maxwellian population are shown in Figure 3, and best fit parameters are given in Table 1.

Table 1

Thermal Model Fit Parameters

\begin{tabular}{|c|c|c|c|c|c|}
\hline Orbit & Date & $\mathrm{L}_{s}$ & Exobase density $\left(10^{6} \mathrm{~cm}^{-3}\right)$ & Exobase Temperature (K) & Escape flux $\left(10^{8} \mathrm{~cm}^{-3} / \mathrm{s}\right)$ \\
\hline 381 & 9 December 2014 & 250 & $1.48_{-0.08}^{+0.09}$ & $179_{-2}^{+2}$ & $2.90_{-0.1}^{+0.1}$ \\
\hline 400 & 13 & 252 & $1.47_{-0.07}^{+0.08}$ & $175_{-2}^{+2}$ & $2.45_{-0.09}^{+0.08}$ \\
\hline 422 & 17 & 254 & $1.37_{-0.07}^{+0.07}$ & $173_{-2}^{+2}$ & $2.06_{-0.07}^{+0.07}$ \\
\hline 460 & 24 & 259 & $1.84_{-0.11}^{+0.13}$ & $161_{-2}^{+2}$ & $1.61_{-0.06}^{+0.07}$ \\
\hline 3604 & 4 August 2016 & 198 & $0.625_{-0.016}^{+0.018}$ & $333_{-6}^{+6}$ & $3.46_{-0.15}^{+0.15}$ \\
\hline 3638 & 10 & 202 & $0.835_{-0.014}^{+0.015}$ & $330_{-4}^{+4}$ & $4.45_{-0.13}^{+0.13}$ \\
\hline 3674 & 17 & 206 & $1.09_{-0.02}^{+0.02}$ & $299_{-3}^{+3}$ & $3.85_{-0.13}^{+0.12}$ \\
\hline 3716 & 25 & 210 & $1.03_{-0.02}^{+0.02}$ & $289_{-3}^{+3}$ & $3.12_{-0.09}^{+0.09}$ \\
\hline
\end{tabular}

Note. For these models, the model scale factor is $0.870 \pm 0.002$, the December 2014 IPH background (back in equation (1) is $626 \pm 23 \mathrm{R}$, and the August 2016 $\mathrm{IPH}$ is $303 \pm 18 \mathrm{R}$. Values given are the median of the sample distribution, with uncertainties recording the distance to the 84th and 16th percentiles. Parameters represent exobase values for a spherically symmetric Maxwellian population of $\mathrm{H}$ atoms. $\mathrm{IPH}=$ interplanetary hydrogen. 

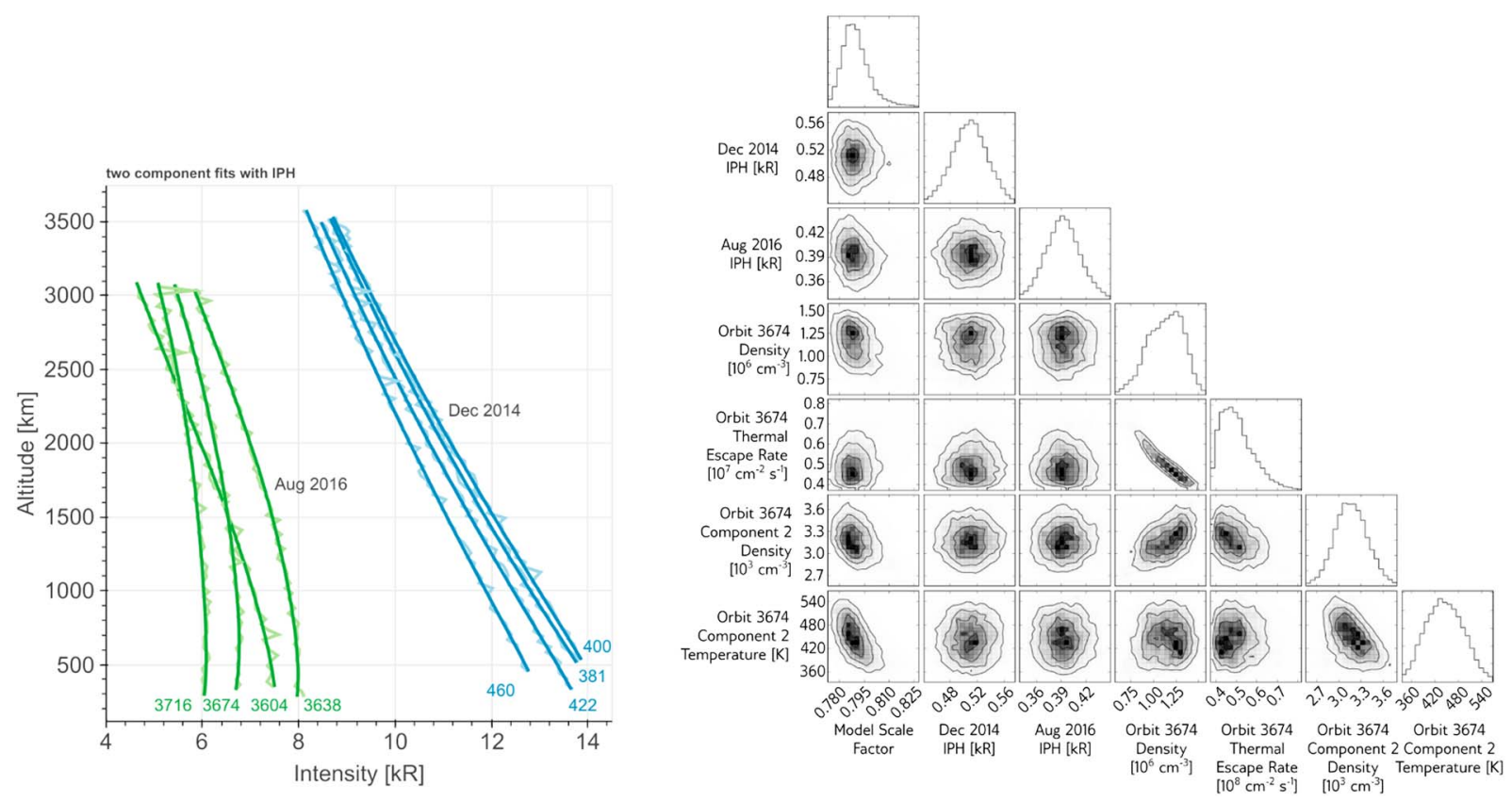

Figure 4. Two-component model best fits. (left) Observed brightness (lighter jagged lines) and best fit two-component models (dark smooth curves). Random uncertainties on data brightness are suppressed for clarity and can be inferred from data scatter or by reference to Figure 2. (right) Posterior distribution of fitted parameters for orbit 3674, as an example of the general covariance of the fitted parameters. In this figure, the $\mathrm{N}$-dimensional probability distribution is visualized via its 2-D marginal distributions in every pair of variables and by the 1-D marginal distributions of each fitted variable. Contours are at 0.5-sigma intervals from 0.5 - to 2 -sigma. Model profiles reproduce the observed brightness very well, eliminating discrepancies exhibited by thermal fits.

The thermal best fits do an adequate job of reproducing the intensity and general shape of the profiles but do not completely reproduce the profile curvature or low-altitude intensities. This indicates that the model can capture the differences in viewing geometry between the profiles and reproduce the gross changes reflected in the profile without the need for an additional population. However, several problems with the best fit profiles and fit parameters indicate the need for an additional population. At the lowest altitudes, the December 2014 model profiles underestimate the intensities, indicating the need for an additional $\mathrm{H}$ population at these altitudes, supplied by coronal transport (and therefore asymmetries in the corona) or the presence of deuterium. In both the December 2014 and August 2016 profiles, the model curvature is different from the data in most cases, indicating the need for an additional model parameter. In addition, the best fit parameters corresponding to these profiles violate a priori expectations, with the December 2014 profiles from later in southern summer reporting lower escape rates than the August 2016 profiles. It is tempting to ascribe this result to interannual variability, but the uncertainty in the IPH background assumed is at fault: The December 2014 observations have a fitted background at the upper end of the allowed range, and the August 2016 observations have a background at the low end, resulting in depressed temperatures and escape rates in December 2014 and enhanced rates in August 2016. Finally, the fitted temperatures for these August 2016 profiles are still high compared to expected thermosphere temperatures from MAVEN (Bougher et al., 2017), providing additional evidence that a more complex model is required to fit the profiles.

Two-component model fits and fit parameters are shown in Figure 4, and best fit parameters are given in Table 2. The thermal and hot components of each fit are shown in Figure D1. This model does a near-perfect job of fitting the data and conforms to more of the a priori expectations than the thermal model. Adding a second population allows the model to more independently fit the upper and lower portions of the data, resulting in no mismatch between the model and data at low altitudes and a near-perfect match to profile curvature. Profiles from December 2014 have rates higher by a factor of 2-4 compared with August 2016, as is expected for later in the Mars year and closer to southern summer solstice. The fitted background $\mathrm{IPH}$ is close to the input prior instead of at extremes, as was the case with the thermal models. Finally, the fitted temperatures are close to those expected based on MAVEN measurements of Ar (Bougher et al., 2017), which was not the case for the single-component thermal fits and lends additional credence to the two-component model. The second component is hotter and far less dense than the thermal component, 
Table 2

Two-Component Model Fit Parameters

\begin{tabular}{|c|c|c|c|c|c|c|c|c|}
\hline Orbit & Date & $L_{s}$ & $\begin{array}{l}\text { Thermal exobase } \\
\text { density }\left(10^{6} \mathrm{~cm}^{-3}\right)\end{array}$ & $\begin{array}{l}\text { Thermal exobase } \\
\text { temperature }(\mathrm{K})\end{array}$ & $\begin{array}{l}\text { Thermal escape } \\
\text { flux }\left(10^{8} \mathrm{~cm}^{-3} / \mathrm{s}\right)\end{array}$ & $\begin{array}{c}\text { Component } 2 \\
\text { density }\left(10^{3} \mathrm{~cm}^{-3}\right)\end{array}$ & $\begin{array}{c}\text { Component } 2 \\
\text { temperature }(\mathrm{K})\end{array}$ & $\begin{array}{c}\text { Total escape } \\
\text { flux }\left(10^{8} \mathrm{~cm}^{-3} / \mathrm{s}\right)\end{array}$ \\
\hline 381 & 9 December 2014 & 250 & $\begin{array}{r}0.77_{-0.10}^{+0.10} \\
\end{array}$ & $231_{-8}^{+9}$ & $7.72_{-0.65}^{+0.64}$ & $3.83_{-0.31}^{+0.30}$ & $408_{-40}^{+41}$ & $\begin{array}{r}8.17_{-0.72}^{+0.69} \\
\end{array}$ \\
\hline 400 & 13 & 252 & $1.86_{-0.23}^{+0.27}$ & $180_{-5}^{+5}$ & $3.88_{-0.09}^{+0.29}$ & $3.28_{-0.37}^{+0.35}$ & $335_{-32}^{+42}$ & $4.07_{-0.33}^{+0.32}$ \\
\hline 422 & 17 & 254 & $\begin{array}{r}1.42_{-0.16}^{+0.19} \\
\text { a }\end{array}$ & $188_{-5}^{+5}$ & $3.93_{-0.07}^{+0.33}$ & $3.74_{-0.30}^{+0.31}$ & $\begin{array}{r}300_{-29}^{+31}\end{array}$ & $4.08_{-0.35}^{+0.36}$ \\
\hline 460 & 24 & 259 & $1.36_{-0.16}^{+0.16}$ & $\begin{array}{r}189_{-5}^{+6} \\
-\end{array}$ & $\begin{array}{r}3.91_{-0.06}^{+0.36} \\
\text { r }\end{array}$ & $4.53_{-0.38}^{+0.37}$ & $\begin{array}{r}328_{-25}^{+26} \\
\text { r }\end{array}$ & $\begin{array}{r}4.15_{-0.37}^{+0.40} \\
\text { - }\end{array}$ \\
\hline 3604 & 4 August 2016 & 198 & $0.113_{-0.008}^{+0.007}$ & $254_{-6}^{+8}$ & $\begin{array}{r}1.89_{-0.15}^{+0.17} \\
\text { - }\end{array}$ & $2.31_{-0.21}^{+0.22}$ & $392_{-44}^{+41}$ & $2.12_{-0.15}^{+0.18}$ \\
\hline 3638 & 10 & 202 & $0.285_{-0.039}^{+0.050}$ & $204_{-10}^{+9}$ & $1.36_{-0.13}^{+0.20}$ & $\begin{array}{r}2.97_{-0.25}^{+0.19} \\
\end{array}$ & $\begin{array}{r}437_{-42}^{+53} \\
-4\end{array}$ & $\begin{array}{r}1.79_{-0.25}^{+0.25} \\
\text { - }\end{array}$ \\
\hline 3674 & 17 & 206 & $1.15_{-0.22}^{+0.18}$ & $148_{-5}^{+9}$ & $0.492_{-0.067}^{+0.096}$ & $\begin{array}{r}3.17_{-0.21}^{+0.23} \\
-0.2\end{array}$ & $444_{-48}^{+52}$ & $0.967_{-0.14}^{+0.15}$ \\
\hline 3716 & 25 & 210 & $0.515_{-0.093}^{+0.116}$ & $\begin{array}{c}177_{-9}^{+10} \\
\end{array}$ & $0.931_{-0.017}^{+0.019}$ & $3.74_{-0.17}^{+0.18}$ & $367_{-24}^{+23}$ & $1.24_{-0.18}^{+0.18}$ \\
\hline
\end{tabular}

Note. For these models, the model scale factor is $0.790_{-0.006}^{+0.009}$, the December $2014 \mathrm{IPH}$ background (back in equation (1) is $510_{-26}^{+26} \mathrm{R}$, and the August $2016 \mathrm{IPH}$ is $392_{-21}^{+21} \mathrm{R}$. Values given are the median of the sample distribution, with uncertainties recording the distance to the 84 th and 16 th percentiles. Parameters represent exobase values for a spherically symmetric Maxwellian population of $\mathrm{H}$ atoms. IPH = interplanetary hydrogen.

contributing 5-25\% of the escaping hydrogen, varying profile-to-profile. Importantly, in our calculations this second $\mathrm{H}$ component makes up a small portion of the total $\mathrm{H}$ density, typically less than $1 \%$, as compared with previous work, which required $10-20 \%$ of the $\mathrm{H}$ present at the exobase to be hot (Bhattacharyya et al., 2015; Chaufray et al., 2008), possibly due to inaccuracies in Mars or solar Lyman alpha brightness. The smaller fraction of energetic $\mathrm{H}$ we infer is more plausible and in closer accordance with the model predictions of a low superthermal $\mathrm{H}$ population (Lichtenegger et al., 2006). The temperature fitted declines in each time period, despite the decreasing Mars-Sun distance. This trend agrees with fitted atmospheric scale heights from IUVS (Bougher et al., 2017) and may reflect short-term cooling imposed on a longer-term seasonal trend for higher temperatures as $L_{s}$ approaches 270 .
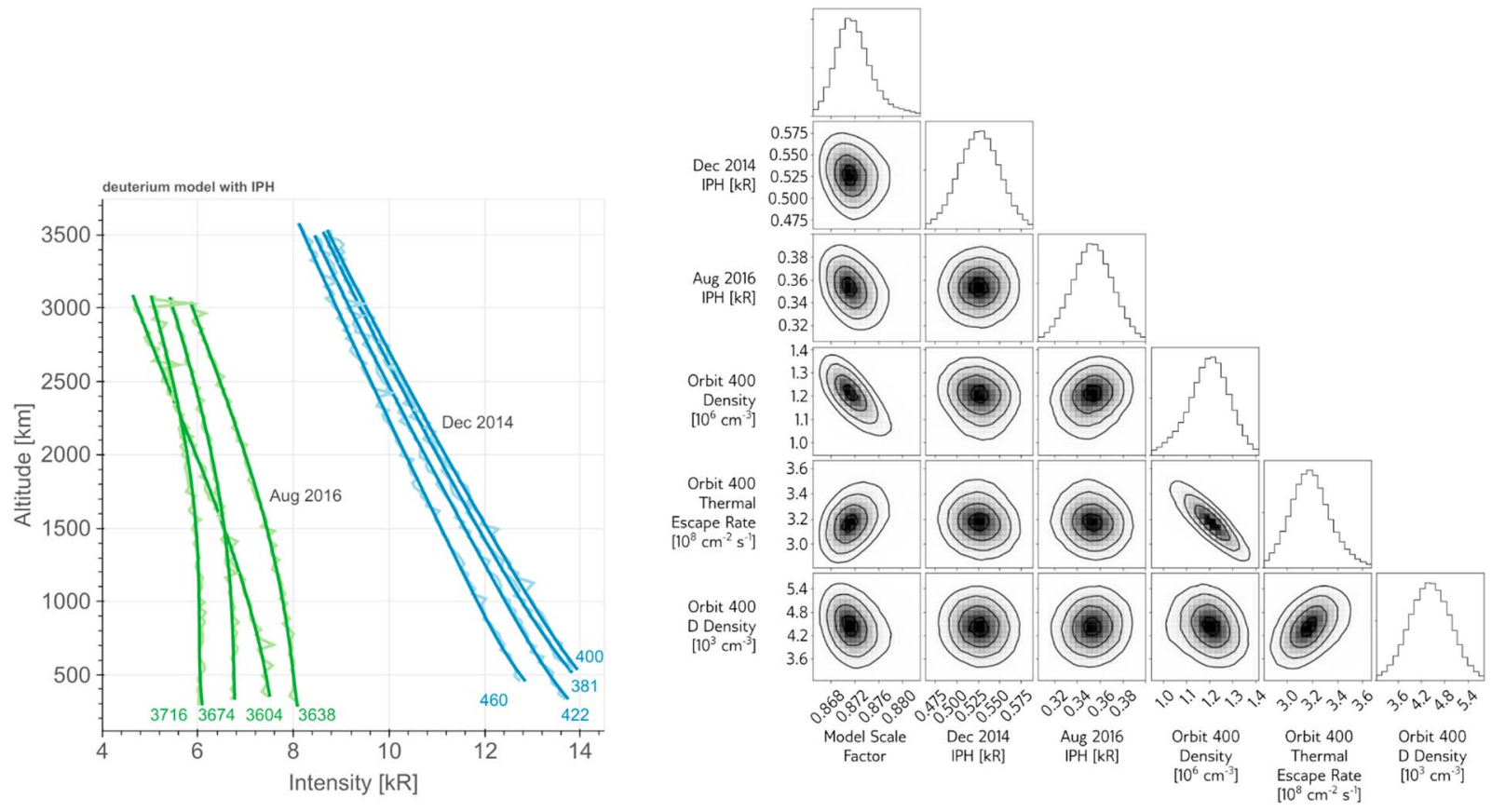

Figure 5. Deuterium model best fits. (left) Observed brightness (lighter jagged lines) and best fit deuterium models (dark smooth curves). Random uncertainties on data brightness are suppressed for clarity and can be inferred from data scatter or by reference to Figure 2. (right) Posterior distribution of fitted parameters for orbit 400, as an example of the general covariance of the fitted parameters. In this figure, the $\mathrm{N}$-dimensional probability distribution is visualized via its 2-D marginal distributions in every pair of variables and by the 1-D marginal distributions of each fitted variable. Contours are at 0.5 -sigma intervals from $0.5-$ to 2-sigma. Model profiles reproduce the observed brightness nearly as well as in the two-component case, demonstrating the degeneracy in models that results in ambiguous escape rates. IPH = interplanetary hydrogen. 
Table 3

Deuterium Model Fit Parameters

\begin{tabular}{|c|c|c|c|c|c|c|}
\hline Orbit & Date & $L_{s}$ & $\begin{array}{l}\text { Thermal exobase } \\
\text { density }\left(10^{6} \mathrm{~cm}^{-3}\right)\end{array}$ & $\begin{array}{l}\text { Thermal exobase } \\
\text { temperature }(\mathrm{K})\end{array}$ & $\begin{array}{l}\text { Thermal escape } \\
\text { flux }\left(10^{8} \mathrm{~cm}^{-3} / \mathrm{s}\right)\end{array}$ & $\begin{array}{l}\text { Deuterium exobase } \\
\text { density }\left(10^{3} \mathrm{~cm}^{-3}\right)\end{array}$ \\
\hline 381 & 9 December 2014 & 250 & $1.09_{-0.096}^{+0.086}$ & $197_{-4}^{+4}$ & $\begin{array}{r}4.09_{-0.21}^{+0.24} \\
\end{array}$ & $4.63_{-0.44}^{+0.45}$ \\
\hline 400 & 13 & 252 & $1.20_{-0.092}^{+0.083}$ & $187_{-3}^{+3}$ & $3.18_{-0.15}^{+0.17}$ & $4.42_{-0.53}^{+0.53}$ \\
\hline 422 & 17 & 254 & $\begin{array}{r}1.07_{-0.075}^{+0.070} \\
-0.07\end{array}$ & $187_{-3}^{+3}$ & $2.83_{-0.12}^{+0.13}$ & $3.56_{-0.29}^{+0.29}$ \\
\hline 460 & 24 & 259 & $1.45_{-0.11}^{+0.096}$ & $173_{-2}^{+3}$ & $2.22_{-0.10}^{+0.12}$ & $6.04_{-0.50}^{+0.51}$ \\
\hline 3604 & 4 August 2016 & 198 & $0.0818_{-0.0062}^{+0.0085}$ & $284_{-13}^{+12}$ & $2.33_{-0.27}^{+0.25}$ & $0.970_{-0.34}^{+0.40}$ \\
\hline 3638 & 10 & 202 & $0.113_{-0.060}^{+0.085}$ & $281_{-10}^{+7}$ & $3.06_{-0.26}^{+0.20}$ & $1.65_{-0.29}^{+0.38}$ \\
\hline 3674 & 17 & 206 & $0.152_{-0.060}^{+0.088}$ & $257_{-7}^{+6}$ & $2.68_{-0.20}^{+0.18}$ & $2.04_{-0.32}^{+0.37}$ \\
\hline 3716 & 25 & 210 & $\begin{array}{r}0.157_{-0.067}^{+0.081} \\
\end{array}$ & $243_{-5}^{+4}$ & $2.06_{-0.12}^{+0.10}$ & $2.89_{-0.26}^{+0.27}$ \\
\hline
\end{tabular}

Note. For these models, the model scale factor is $0.871_{-0.003}^{+0.003}$, the December $2014 \mathrm{IPH}$ background (back in equation (1) is $526_{-24}^{+24} \mathrm{R}$, and the August $2016 \mathrm{IPH}$ is $353_{-18}^{+18} R$. Values given are the median of the sample distribution, with uncertainties recording the distance to the 84 th and 16 th percentiles. Parameters represent exobase values for a spherically symmetric Maxwellian population of $\mathrm{H}$ atoms.

Deuterium model fits and fit parameters are shown in Figure 5 and Table 3. The H and D components of each fit are shown in Figure D2. These fits are slightly worse than the two-component fits, particularly in fitting the curvature at the bottom of the August 2016 profiles and in slightly overestimating the brightness at the lowest altitudes of the December 2014 profiles. A comparison of all three model fits and the data is given in Figures D3 and D4. Deuterium exobase temperatures are higher than for the two-component model; this is most noticeable in August of 2016. As with the two-component model, the IPH background is in close alignment with the prior. A seasonal trend is visible in the $\mathrm{H}$ escape flux and in the $\mathrm{D}$ abundance required at the exobase, both of which are enhanced closer to southern summer solstice. In both seasons the $D$ fraction required at the exobase is on the order of $10^{-3}-10^{-2}$, exceeding model predictions by Yung et al. (1988) and Krasnopolsky (2002) by a factor of 10-100, possibly indicating much larger transport of $D$ to the upper atmosphere than predicted. However, these model predictions predate MAVEN and Mars Express observations of the upper atmosphere and include high thermospheric temperatures that exceed MAVEN-measured values, potentially depressing predicted $\mathrm{D}$ abundances and motivating future model investigation. Elevated deuterium abundances have been found in southern summer using the echelle channel of IUVS (Clarke et al., 2017) and have been indicated in some low-resolution observations (Chaufray et al., 2017), adding support to notion that more $\mathrm{D}$ could be present than would otherwise be expected.

Degeneracies in the model parameter space are more fully explored by our Monte Carlo techniques, so that the interplay of instrument calibration, density, and temperature can be understood. We find that changes in the instrument calibration have a disproportionate effect on the fitted number density and temperature, as is expected for an optically thick emission. For example, a 1\% change in the calibration of the instrument results in 10-20\% anticorrelated changes in the fitted escape flux and exobase number density for each profile and model, as can be seen in Figures 3-5. Precise knowledge of the instrument calibration is therefore required in order to extract escape fluxes and densities from an optically thick emission in the absence of additional information that can break parameter degeneracies. Supply of this information from thermospheric climatology, distant optically thin regions of the corona, or some other source would moderate the ability of calibration to affect derived escape rates.

The required model scale factor for all cases is less than 1.0, indicating that the model output needs to be scaled down in order to match the data. The implication is that MAVEN IUVS brightnesses are too dim, and the instrument is less sensitive at Lyman alpha by $10-20 \%$ compared to the current calibration. Because the Lyman alpha calibration of IUVS is only known to perhaps 20\% (Mayyasi et al., 2017), this conclusion is not in conflict with prior analysis. Early indications that the instrument brightnesses were too large (Chaffin et al., 2015) resulted from multiple effects: (1) uncertain calibration of the input solar flux from EUVM, (2) fitting in number density-temperature space rather than number density-escape flux space (as explained in Appendix C), and (3) fitting a calibration factor solely to observations from December 2014 rather than to multiple time 
periods with multiple scan geometries. The additional time period included in the present analysis adds a new range of solar flux conditions and observing geometries to provide additional leverage on the systematic calibration offset of the instrument.

For each model, the thermal escape rates are within the range of previously derived values. Anderson and Hord (1971) found a rate of $1.8 \times 10^{8} \mathrm{~cm}^{-2} / \mathrm{s}$, Chaufray et al. (2008) found $1.4 \pm 0.6 \times 10^{8} \mathrm{~cm}^{-2} / \mathrm{s}$, and Feldman et al. (2011) found $1.3 \times 10^{8} \mathrm{~cm}^{-2} / \mathrm{s}$. Chaffin et al. (2014) and Bhattacharyya et al. (2015) found a range of escape rates from 0.1 to $50 \times 10^{8} \mathrm{~cm}^{-2} / \mathrm{s}$, depending on the assumed model temperature and whether a nonthermal component was included in the analysis. We note that while these previous analyses obtained similar best fit parameters, the quality of our profile fits is improved relative to these earlier studies, particularly in matching the curvature of the profile and matching the mean intensity at both high and low altitudes. In particular, our two-component model profiles reproduce the shape of the data with reasonable fit densities and temperatures - likely the consequence of our more complete exploration of the full parameter space without ad hoc assumptions and the inclusion of a simultaneous fit parameter for the instrument absolute calibration.

Our two-component fits are consistent with previously observed seasonal trends in $\mathrm{H}$ escape. Both the two-component and deuterium model fits exhibit 2-3 times higher escape rates in December 2014 at $L_{s} \sim$ 250 than in August 2016 at $L_{s} \sim 200$, suggesting a rapid uptick approaching southern summer solstice reported using in situ measurements (Halekas, 2017).

\section{Prospects for Future Progress}

Our results produce near-perfect fits to brightness profiles for two different assumed exobase populations, a two-component $\mathrm{H}$ fit and a fit assuming the presence of $\mathrm{D}$ in the corona. Exploring and resolving this degeneracy will require additional information from other observations of the Mars corona and thermosphere. Models can also provide information useful in establishing the prior probability of various $\mathrm{H}$ populations. Finally, higher fidelity in retrieval models will be required to place accurate limits on the $\mathrm{H}$ population in the presence of non-Maxwellian velocity distributions.

Additional MAVEN data will aid in selection of an appropriate model to infer $\mathrm{H}$ escape rates. MAVEN IUVS contains an echelle channel designed to resolve deuterium Lyman alpha from the $\mathrm{H}$ line, which has been used to observe variability in thermospheric D brightness (Clarke et al., 2017; Mayyasi et al., 2017). These brightnesses could be used to constrain the thermospheric $D$ population as a prior on coronal $H$ retrievals. The echelle can also provide a direct measurement of the IPH brightness, potentially allowing the use of a more restrictive prior on IPH brightnesses that could bring thermal fits into closer alignment with our two component and deuterium results. While MAVEN makes no in situ measurement of hydrogen densities, thermospheric temperatures can be extracted from the mass spectrometer (Mahaffy et al., 2014) and IUVS measurements on most periapsis passes (Bougher et al., 2017), providing a point measurement of the temperature on the opposite side of the planet from the coronal scans. IUVS limb scans made on the outbound segment of the orbit provide information on the thermospheric scale height and temperature beneath the coronal scans, which could also be used as a prior on thermal $\mathrm{H}$ temperatures. MAVEN's EUVM can also measure $\mathrm{CO}_{2}$ scale height temperatures from solar occultations in certain orbital configurations (Thiemann et al., 2018). Combining these measurements could yield information about temperature anisotropy around the planet useful in forward modeling of atmospheric brightness, potentially replacing the analytic temperature profile from Krasnopolsky (2002) used in the current work. Finally, MAVEN SWIA measurements have be used to derive the column density of $\mathrm{H}$ upstream of the bow shock, which could be used to providing an additional constraint on the allowed H population (Halekas et al., 2013; Halekas, 2017). Incorporation of additional constraints might provide enough information to robustly perform three-component fits to the $\mathrm{H}$ distribution, fitting thermal and nonthermal $\mathrm{H}$ as well as deuterium.

Physical and chemical modeling would also provide prior information on the expected H and D abundance and velocity distribution. Photochemical models of deuterium abundance in the upper atmosphere (Krasnopolsky, 2002; Yung et al., 1988) predate MAVEN measurements of thermospheric temperature and other properties and should be updated to provide more secure priors on the expected abundance of deuterium at the exobase. In addition, updated kinetic models of the upper atmosphere that simulate the $\mathrm{H}$ 
velocity distribution and the production of hot $\mathrm{H}$ could be used to inform the selection of the input velocity distribution used in coronal brightness models. The vast majority of the $\mathrm{H}$ observed in Lyman alpha is bound to Mars; determining the escape rate from this bound component depends on precise knowledge of the relationship between the thermal core of the velocity distribution and the high-energy escaping tail.

Finally, retrieval models must be updated to relax assumptions about the structure of the corona. First, calculations of the coronal density and Lyman alpha scattering should be made to self-consistently incorporate non-Maxwellian velocity distributions with enhanced or depleted thermal tails. Performing these calculations will require relaxing the assumption of Gaussian line shapes in the corona and may require a more accurate treatment of frequency redistribution. Second, physical and radiative transfer forward models should be modified to include departures from spherical symmetry, including a noon-midnight temperature contrast. These updated models will be required to interpret 2-D maps of the corona that sample many local times, such as those provided by MAVEN's apoapsis images (Chaffin et al., 2015) and the Hubble Space Telescope (Bhattacharyya et al., 2015; Clarke et al., 2014).

\section{Appendix A: All Coronal Profiles From the Study Period}

All coronal profiles from the study period are shown in Figure A1. Because the solar Lyman alpha brightness is observed by the MAVEN Extreme Ultraviolet Monitor to vary by $\pm 10 \%$ throughout each time period as a result of solar rotation, we normalize the profiles in this figure, scaling them to the input solar brightness observed for the first profile to reveal the changes due to the evolving corona and observation geometry. The profiles we select for this study span the time periods of interest and capture the range of observed profile shapes.

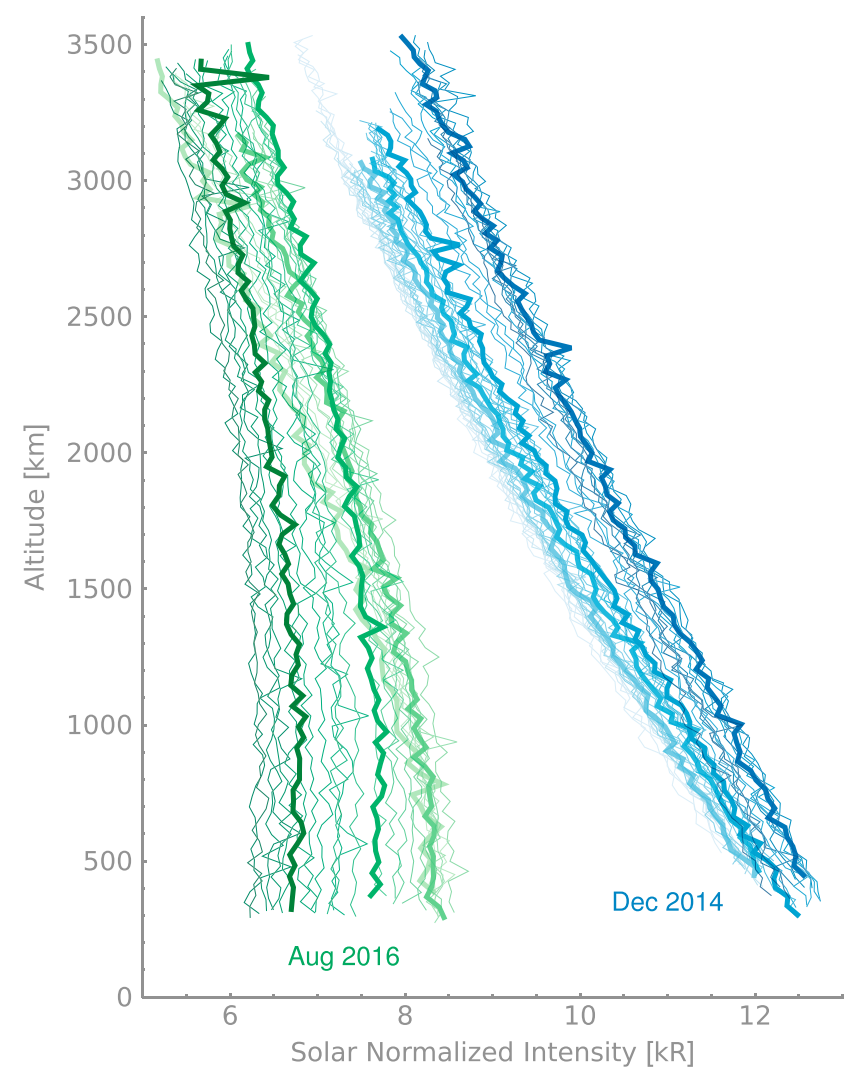

Figure A1. Observed coronal profiles for December 2014 and August 2016. Thin lines show all observed profiles, with colors shading from light to dark within each month. The observed brightness for each orbit has been normalized to the same input solar brightness using MAVEN/EUVM observations, to clarify nonsolar variations. Thick lines show orbits selected for further study in this work. The selected orbits span the available data and constitute a representative sample of the observed profile shapes. MAVEN = Mars Atmosphere and Volatile Evolution; EUVM = Extreme Ultraviolet Monitor. 


\section{Appendix B: Absolute Calibration of IUVS at Lyman Alpha}

The IUVS far ultraviolet calibration is a compromise between stellar observations and models of the Mars $\mathrm{H}$ corona. IUVS was calibrated during the cruise to Mars through observation of ultraviolet reference stars, including $\beta$ CMa, $\beta$ Cen, $\alpha$ Cru, and $\alpha$ Vir. Reference spectra from SOLSTICE were used to calibrate the instrument (see; Snow et al., 2013). Because Lyman alpha from these stars is extincted by interstellar hydrogen, the calibration at Lyman alpha is determined by interpolation of the instrument sensitivity from measurements on both sides of the line. Upon arrival at Mars, comparison of the observed Lyman alpha brightness of the Mars corona with previous observations and model expectations suggested that IUVS intensities were too bright, and IUVS intensities were reduced by a factor of 1.27, while simultaneously setting the systematic calibration uncertainty of IUVS at $25 \%$. This systematic uncertainty is larger than that carried by SOLSTICE (McClintock et al., 2005), TIMED/SEE (SEE; Woods, 2005) and HST/STIS (Riley, 2017), which all carry systematic uncertainties near 5\% (1-sigma) at Lyman alpha. Our results indicate that IUVS Lyman alpha profiles are best fit by a 10-20\% increase in IUVS Lyman alpha intensities, which would partially undo the compromise calibration imposed early in the mission. This modification to the uncertainties would not greatly affect the analysis of Mayyasi et al. (2017), which found that IUVS Lyman alpha observations of the IPH agreed with other instruments at about the $10 \%$ level.

\section{Appendix C: Effect of Interpolation and Parameterization on Retrieved Temperatures}

Radiative transfer calculations are computationally expensive. As a result, many authors (Bhattacharyya et al., 2015; Chaffin et al., 2014; Chaufray et al., 2008) have employed interpolation schemes to reduce computation time. These schemes sample parameter space on a grid and precompute solutions there, interpolating these solutions to produce values at intermediate points. In addition to saving time when modeling an individual observation, this scheme also enables scattering source functions to be precomputed ahead of time for all observations. These gridded source functions are then integrated along the instrument line of sight for each observation. Interpolation is not detrimental to the solution as long as interpolation points are chosen sufficiently close together to capture the variations in the merit function (chi-squared or log-likelihood). Capturing the structure of the interpolated merit function necessarily includes reproducing any degeneracies present.

There exists an inherent degeneracy in fitting an optically thick emission such as Mars $\mathrm{H}$ Lyman alpha. Brightness profiles can be interpreted as resulting from a large column density of $\mathrm{H}$ atoms at a low temperature or a

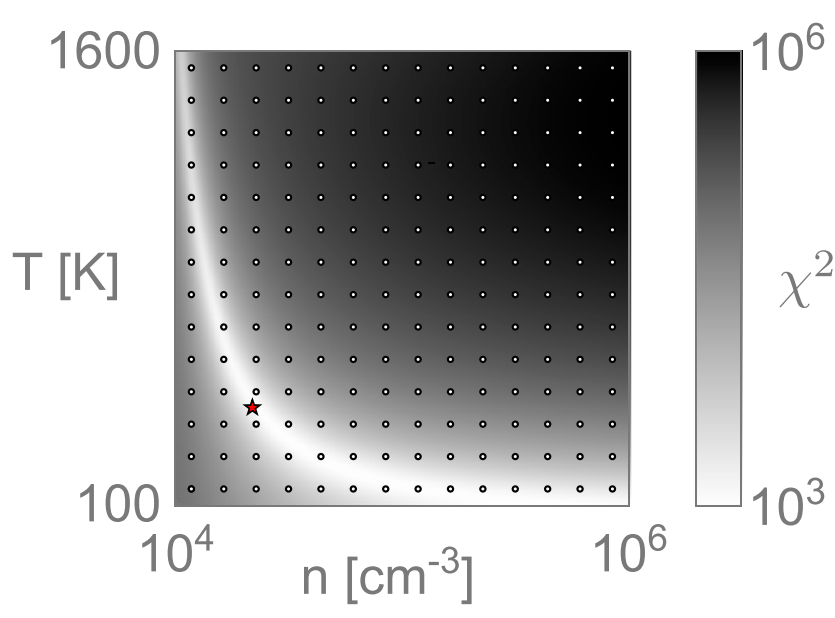

Figure C1. Interaction of grid sampling and parameter degeneracy. Parameterization in number density and temperature produces a nonlinear degeneracy, where acceptable fits are arrayed along an approximately hyperbolic track (light region of shading). Rectangular grid sampling at a relatively coarse resolution (white points) produces points that are more likely to intersect the array of acceptable fits at high temperatures and low densities, missing the true minimum that lies in between grid points (red star). smaller column density of $\mathrm{H}$ atoms at a higher temperature. This degeneracy is fundamental to the problem. An increase in Mars $\mathrm{H}$ temperature pushes atoms into the optically thin line wings. Because the solar line is broad relative to the planetary line, atoms at the extremes of the velocity distribution outside the line core still scatter light, having a disproportionate effect on observed intensities. Thus, a small increase in exospheric temperature can replicate the effect of a large increase in the number of $\mathrm{H}$ atoms along the line of sight. This problem is not solved by examination of the shape of the altitude profile, which in an optically thin case is used to extract the atmospheric temperature from the intensity scale height. Outside information is required to break the degeneracy, or else the emission must be tracked to a large enough altitude that it becomes optically thin. In the case of MAVEN, the $\mathrm{H}$ temperature or density is not measured directly by any instrument, and the orbit is firmly embedded in the optically thick corona. We could assume $\mathrm{H}$ is isothermal with $\mathrm{CO}_{2}$ or $\mathrm{Ar}$ temperatures measured by MAVEN, or make assumptions based on modeling work (e.g.Terada et al., 2016) to derive an $\mathrm{H}$ temperature from these, but we defer this to future work. In any case, nonthermal processes and the effect of Jeans escape may produce a different temperature in $\mathrm{H}$ than in other species.

Interplay between the degeneracy inherent in fitting an optically thick emission and the interpolation grid may lead to artificially high or low 
temperatures in the resulting fit. Figure $\mathrm{C} 1$ shows a schematic illustration of the problem. When expressed in number density and temperature, fits to a particular orbit exhibit a band of acceptable fits that tracks from high $\mathrm{T} /$ low $\mathrm{n}$ to low $\mathrm{T} / \mathrm{high} \mathrm{n}$ with a hyperbolic shape. Reasonable densities and temperatures lie near the vertex, and the best fit is likely near this point. However, on an interpolated grid the best fit is not necessarily captured by any of the grid points. Instead, points near the optimum may be sampled and the true best fit missed. In these cases a naive optimization returns the most optimal grid point, nearest to the center of the band of degenerate acceptable fits. However, this point is not necessarily near the best fit, and the interaction of the grid and the structure of the merit function can lead optimizers astray. At the low density end of the degeneracy, the slope of the band becomes steep, moving slowly through densities. For a rectilinear interpolation grid, this steep slope has a far greater chance of passing near an interpolation point, which correspondingly increases the chance of a naive optimizer finding the best grid point at high temperature and low density. Similarly, at high densities, the band crosses temperatures slowly, and a high density-low temperature grid point could also be preferred to the true best fit, which could more easily avoid the grid. We believe that this phenomenon may drive the high temperatures retrieved by previous authors, including Bhattacharyya et al. (2015), Chaffin et al. (2014), Chaufray et al. (2008), and others, all of which use optimization on an interpolation grid.

A different parameterization can better avoid spurious best grid points, resulting in optimal grid points closer to the true best fit. Expressed in number density-escape flux space, the degeneracy is linear rather than hyperbolic, so that all grid points have an equal chance of interacting with the degeneracy the same way. With finely spaced grid points, points adjacent to the true best fit are likely to be selected in optimization. This diminished bias is the reason we perform our fits in number density-escape flux space rather than number density-temperature space. In tests using number density-temperature space instead, we find that even the MCMC technique prefers high-temperature fits. By contrast, in our preferred parameterization the retrieved temperatures are in closer alignment with expected neutral temperatures.

\section{Appendix D: Additional Model/Data Comparison Plots}

Additional plots comparing model fits to the data are shown below.

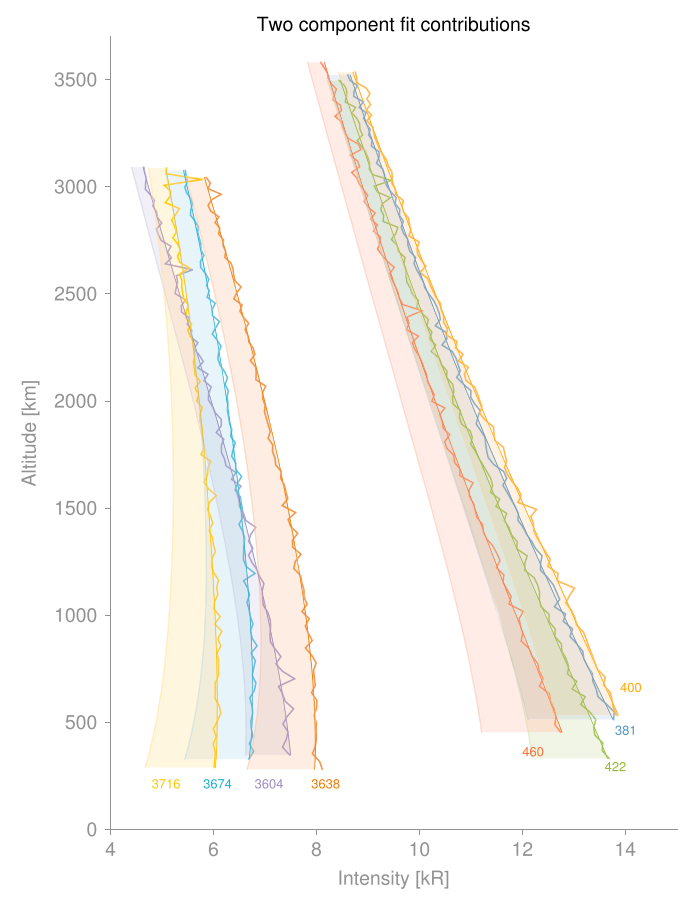

Figure D1. Model/data comparison for two-component fits. Jagged lines show data for each orbit. Thermal+Hot two-component model fits are the smooth curves that pass through the data. The thermal $\mathrm{H}$ population alone is shown as the lower brightness curve to the left of each complete fit, with the difference made up by the fitted hot component. 


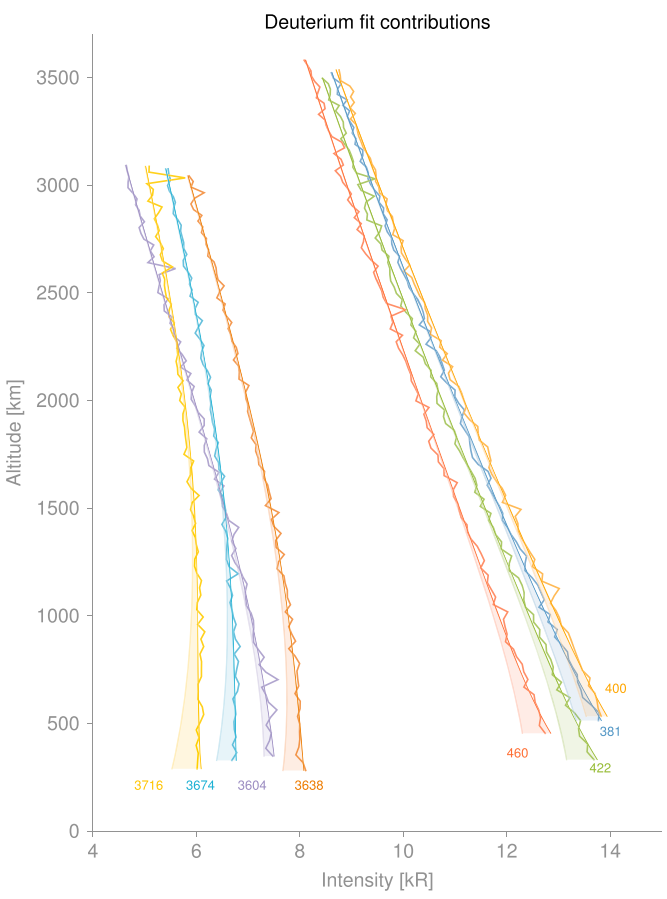

Figure D2. Model/data comparison for deuterium fits. Jagged lines show data for each orbit. H+D model fits are the smooth curves that pass through the data. The thermal $\mathrm{H}$ population alone is shown as the lower brightness curve to the left of each complete fit, with the difference made up by the fitted deuterium component.
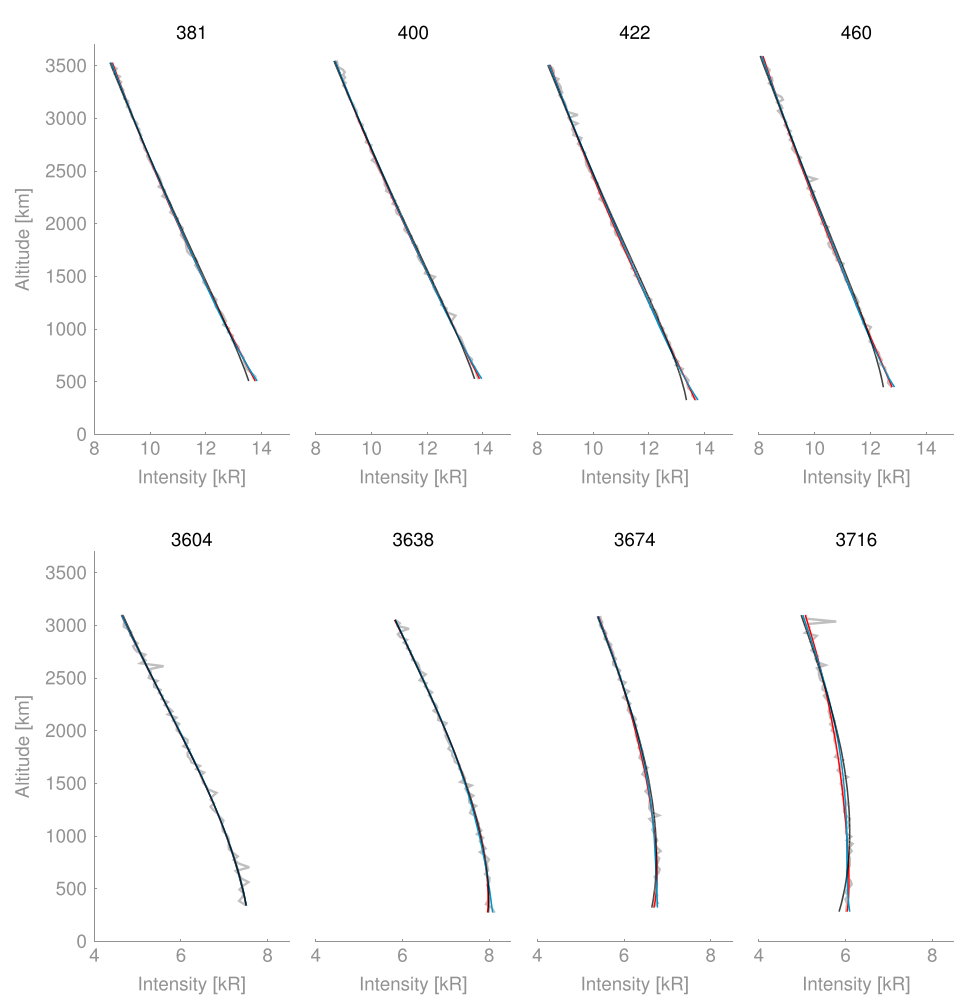

Figure D3. Comparison of three model fitting techniques and data. Each orbit subpanel shows the data (gray), thermal model (black), two-component model (red), and deuterium model (blue). 

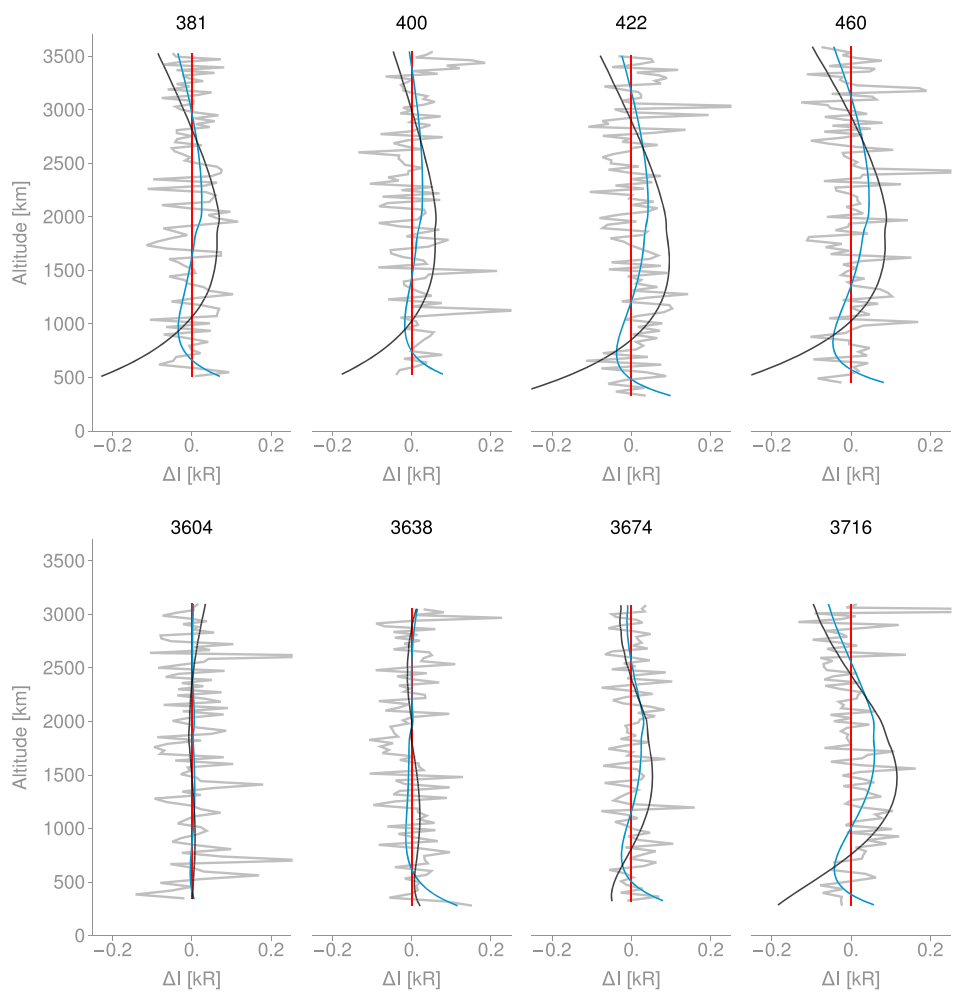

Figure D4. Differenced comparison of three model fitting techniques and data. Data and models are presented as differences from the two-component model fit. Each orbit subpanel shows the data (gray), thermal model (black), two-component model (red), and deuterium model (blue).

\section{Acknowledgments}

IUVS data are available on the Planetary Data System at http://atmos.nmsu.edu/ data_and_services/atmospheres_data/ MAVEN/maven_iuvs.html. We begin our processing with v06 data, but v07 or later products will also work as our procedure begins with instrument counts and is insensitive to the absolute calibration. All coauthors were supported by the MAVEN mission. M. S. Chaffin was supported in part by NASA MDAP grant 16-MDAP16_2-0159. J.- Y. Chaufray and F. Montmessin are funded by the program "Systeme Solaire" of Centre National d'Etudes Spatiales. A. Stiepen is supported by the Fund for Scientific Research (F.R.S.-FNRS). This work utilized the RMACC Summit supercomputer, which is supported by the National Science Foundation (awards ACl-1532235 and ACl-1532236), the University of Colorado Boulder, and Colorado State University. The Summit supercomputer is a joint effort of the University of Colorado Boulder and Colorado State University.

\section{References}

Anderson, Jr. D. E. (1974). Mariner 6, 7, and 9 ultraviolet spectrometer experiment: Analysis of hydrogen Lyman alpha data. Journal Geophysical Research, 79, 1513-1518. https://doi.org/10.1029/JA079i010p01513

Anderson, Jr. D. E., \& Hord, C. W. (1971). Mariner 6 and 7 ultraviolet spectrometer experiment: Analysis of hydrogen Lyman-alpha data. Journal Geophysical Research, 76, 6666-6673. https://doi.org/10.1029/JA076i028p06666

Anderson, D. E., \& Hord, C. W. (1977). Multidimensional radiative transfer: Applications to planetary coronae. Planetary and Space Science, 25(6), 563-571. https://doi.org/10.1016/0032-0633(77)90063-0

Babichenko, S. I., Deregusov, E. V., Kurt, V. G., Romanova, N. N., Skliankin, V. A., Smirnov, A. S., et al. (1977). Measurements in interplanetary space and in the martian upper atmosphere with a hydrogen absorption-cell spectrophotometer for l-alpha radiation on-board Mars 4-7 spaceprobes. Space Science Instrumentation, 3, 271-286.

Bertaux, J. L., Kyrölä, E., Quémerais, E., Pellinen, R., Lallement, R., Schmidt, W., et al. (1995). SWAN: A study of solar wind anisotropies on SOHO with Lyman alpha sky mapping. Solar Physics, 1-2, 403-439. https://doi.org/10.1007/bf00733435

Bertucci, C., Romanelli, N., Chaufray, J. Y., Gomez, D., Mazelle, C., Delva, M., et al. (2013). Temporal variability of waves at the proton cyclotron frequency upstream from mars: Implications for mars distant hydrogen exosphere. Geophysical Research Letters, 40, 3809-3813. https://doi.org/10.1002/grl.50709

Bhattacharyya, D., Clarke, J. T., Bertaux, J. L., Chaufray, J. Y., \& Mayyasi, M. (2015). A strong seasonal dependence in the Martian hydrogen exosphere. Geophysical Research Letters, 42, 8678-8685. https://doi.org/10.1002/2015gl065804

Bhattacharyya, D., Clarke, J., Bertaux, J. L., Chaufray, J. Y., \& Mayyasi, M. (2017). Analysis and modeling of remote observations of the Martian hydrogen exosphere. Icarus, 281, 264-280. https://doi.org/10.1016/j.icarus.2016.08.034

Bougher, S. W., Roeten, K. J., Olsen, K., Mahaffy, P. R., Benna, M., Elrod, M., et al. (2017). The structure and variability of mars dayside thermosphere from MAVEN NGIMS and IUVS measurements: Seasonal and solar activity trends in scale heights and temperatures. Journal of Geophysical Research: Space Physics, 122, 1296-1313. https://doi.org/10.1002/2016JA023454

Brooks, S. P., \& Gelman, A. (1998). General methods for monitoring convergence of iterative simulations. Journal of Computational and Graphical Statistics, 7(4), 434. https://doi.org/10.2307/1390675

Chaffin, M. S., Chaufray, J. Y., Deighan, J., Schneider, N. M., McClintock, W. E., Stewart, A. I. F., et al. (2017). Elevated atmospheric escape of atomic hydrogen from Mars induced by high-altitude water. Nature Geoscience, 10, 174-178. https://doi.org/10.1038/ngeo2887

Chaffin, M. S., Chaufray, J. Y., McClintock, W. E., Thiemann, E., Clarke, J. T., Holsclaw, G. M., et al. (2015). Three-dimensional structure in the Mars H corona revealed by IUVS on MAVEN. Geophysical Research Letters, 42, 9001-9008. https://doi.org/10.1002/2015gl065287

Chaffin, M. S., Chaufray, J. Y., Stewart, I., Montmessin, F., Schneider, N. M., \& Bertaux, J. L. (2014). Unexpected variability of Martian hydrogen escape. Geophysical Research Letter, 41,314-320. https://doi.org/10.1002/2013gl058578

Chamberlain, J. W (1963). Planetary coronae and atmospheric evaporation. Planetary and Space Science, 11, 901. https://doi.org/10.1016/0032-0633(63)90122-3

Chaufray, J. Y., Bertaux, J. L., Leblanc, F., \& Quémerais, E. (2008). Observation of the hydrogen corona with SPICAM on Mars express. Icarus, 195, 598-613. https://doi.org/10.1016/j.Icarus.2008.01.009 
Chaufray, J., Chaffin, M., Clarke, J., Deighan, J., Mayyasi, M., Jain, S., et al. (2017). Possible observations of deuterium Lyman-alpha emission with the low-resolution mode of MAVEN/IUVS. In International Conference on Mars Aeronomy. Boulder, CO.

Clarke, J. T., Bertaux, J. L., Chaufray, J. Y., Gladstone, G. R., Quemerais, E., Wilson, J. K., \& Bhattacharyya, D. (2014). A rapid decrease of the hydrogen corona of Mars. Geophysical Research Letter, 41, 8013-8020. https://doi.org/10.1002/2014GL061803

Clarke, J. T., Mayyasi, M., Bhattacharyya, D., Schneider, N. M., McClintock, W. E., Deighan, J. I., et al. (2017). Variability of D and H in the Martian upper atmosphere observed with the MAVEN IUVS echelle channel. Journal of Geophysical Research: Space Physics, 122, $2336-2344$. https://doi.org/10.1002/2016JA023479

Deighan, J., Chaffin, M. S., Chaufray, J. Y., Stewart, A. I. F., Schneider, N. M., Jain, S. K., et al. (2015). MAVEN IUVS observation of the hot oxygen corona at Mars. Geophysical Research Letters, 42, 9009-9014. https://doi.org/10.1002/2015GL065487

Dostovalov, S. B., \& Chuvakhin, S. D. (1973). On the distribution of neutral hydrogen in the upper atmosphere of Mars. Cosmic Research, $11,687$.

Dubinin, E., Fraenz, M., Woch, J., Barabash, S., Lundin, R., \& Yamauchi, M. (2006). Hydrogen exosphere at Mars: Pickup protons and their acceleration at the bow shock. Geophysical Research Letter, 33, L22103. https://doi.org/10.1029/2006GL027799

Emerich, C., Lemaire, P., Vial, J. C., Curdt, W., Schühle, U., \& Wilhelm, K. (2005). A new relation between the central spectral solar H I Lyman $\alpha$ irradiance and the line irradiance measured by SUMER/SOHO during the cycle 23. Icarus, 178, 429-433. https://doi.org/10.1016/j.lcarus.2005.05.002

Eparvier, F. G., Chamberlin, P. C., Woods, T. N., \& Thiemann, E. M. B. (2015). The solar Extreme Ultraviolet Monitor for MAVEN. Space Science Reviews, 195, 293-301. https://doi.org/10.1007/s11214-015-0195-2

Fedorova, A., Bertaux, J. L., Betsis, D., Montmessin, F., Korablev, O., Maltagliati, L., \& Clarke, J. (2018). Water vapor in the middle atmosphere of Mars during the 2007 global dust storm. Icarus, 300, 440-457. https://doi.org/10.1016/j.icarus.2017.09.025

Feldman, P. D., Steffl, A. J., Parker, J. W., A'Hearn, M. F., Bertaux, J. L., et al. (2011). Rosetta -Alice observations of exospheric hydrogen and oxygen on Mars. Icarus, 214, 394-399. https://doi.org/10.1016/j.lcarus.2011.06.013

Foreman, M. D., Hogg, D. W., Lang, D., \& Goodman, J. (2013). emcee: The MCMC hammer. Publications of the Astronomical Society of the Pacific, 125(925), 306-312. https://doi.org/10.1086/670067

Futaana, Y., Barabash, S., Grigoriev, A., Holmström, M., Kallio, E., Brandt, P. C., et al. (2006). First ENA observations at Mars: ENA emissions from the Martian upper atmosphere. Icarus, 182, 424-430. https://doi.org/10.1016/j.icarus.2005.09.019

Gelman, A., \& Rubin, D. B. (1992). Inference from iterative simulation using multiple sequences. Statistical Science, 7(4), $457-472$. https://doi.org/10.1214/ss/1177011136

Gunell, H., Brinkfeldt, K., Holmström, M., Brandt, P. C., Barabash, S., et al. (2006). First ENA observations at Mars: Charge exchange ENAs produced in the magnetosheath. Icarus, 182, 431-438. https://doi.org/10.1016/j.icarus.2005.10.027

Halekas, J. S. (2017). Seasonal variability of the hydrogen exosphere of Mars. Journal of Geophysical Research: Planets, 122, $901-911$. https://doi.org/10.1002/2017JE005306

Halekas, J. S., Lillis, R. J., Mitchell, D. L., Cravens, T. E., Mazelle, C., Connerney, J. E. P., et al. (2015). Maven observations of solar wind hydrogen deposition in the atmosphere of Mars. Geophysical Research Letter, 42, 8901-8909. https://doi.org/10.1002/2015GL064693

Halekas, J. S., Ruhunusiri, S., Harada, Y., Collinson, G., Mitchell, D. L., Mazelle, C., et al. (2017). Structure, dynamics, and seasonal variability of the Mars-solar wind interaction: MAVEN solar wind ion analyzer in-flight performance and science results. Journal of Geophysical Research: Space Physics, 122, 547-578. https://doi.org/10.1002/2016JA023167

Halekas, J. S., Taylor, E. R., Dalton, G., Johnson, G., Curtis, D. W., McFadden, J. P., et al. (2013). The solar wind ion analyzer for MAVEN. Space Science Reviews, 195(1-4), 125-151. https://doi.org/10.1007/s11214-013-0029-z

Heavens, N. G., Kleinböhl, A., Chaffin, M. S., Halekas, J. S., Kass, D. M., Hayne, P. O., et al. (2018). Hydrogen escape from mars enhanced by deep convection in dust storms. Nature Astronomy, 2(2), 126-132. https://doi.org/10.1038/s41550-017-0353-4

Jakosky, B. M., Lin, R. P., Grebowsky, J. M., Luhmann, J. G., Mitchell, D. F., Beutelschies, G., et al. (2015). The Mars Atmosphere and Volatile Evolution (MAVEN) mission. Space Science Reviews, 195, 3-48. https://doi.org/10.1007/s11214-015-0139-x

Krasnopolsky, V. A. (2002). Mars' upper atmosphere and ionosphere at low, medium, and high solar activities: Implications for evolution of water. Journal Geophysical Research, 107, 5128. https://doi.org/10.1029/2001JE001809

Krasnopolsky, V. A. (2015). Variations of the $\mathrm{HDO} / \mathrm{H}_{2} \mathrm{O}$ ratio in the Martian atmosphere and loss of water from Mars. Icarus, 257, $377-386$. https://doi.org/10.1016/j.icarus.2015.05.021

Lallement, R., Bertaux, J. L., \& Dalaudier, F. (1985). Interplanetary Lyman-alpha spectral profiles and intensities for both repulsive and attractive solar force fields: Predicted absorption pattern by a hydrogen cell. Astronomy \& Astrophysics, 150, 21 - 32.

Lichtenegger, H. I. M., Lammer, H., Kulikov, Y. N., Kazeminejad, S., Molina-Cuberos, G. H., Rodrigo, R., et al. (2006). Effects of low energetic neutral atoms on Martian and Venusian dayside exospheric temperature estimations. Space Science Review, 126, 469-501. https://doi.org/10.1007/s11214-006-9082-1

Mahaffy, P. R., Benna, M., King, T., Harpold, D. N., Arvey, R., Barciniak, M., et al. (2014). The neutral gas and ion mass spectrometer on the Mars Atmosphere and Volatile Evolution mission. Space Science Reviews, 195(1-4), 49-73. https://doi.org/10.1007/s11214-014-0091-1

Maltagliati, L., Montmessin, F., Korablev, O., Fedorova, A., Forget, F., Määttänen, A., et al. (2013). Annual survey of water vapor vertical distribution and water-aerosol coupling in the Martian atmosphere observed by SPICAM/MEx solar occultations. Icarus, 223(2), 942-962. https://doi.org/10.1016/j.icarus.2012.12.012

Mayyasi, M., Clarke, J., Bhattacharyya, D., Deighan, J., Jain, S., Chaffin, M., et al. (2017). The variability of atmospheric deuterium brightness at Mars: Evidence for seasonal dependence. Journal of Geophysical Research: Space Physics, 122, 10,811-10,823. https://doi.org/10.1002/2017JA024666

Mayyasi, M., Clarke, J., Quémerais, E., Katushkina, O., Bhattacharyya, D., Chaufray, J. Y., et al. (2017). IUVS echelle-mode observations of interplanetary hydrogen: Standard for calibration and reference for cavity variations between Earth and Mars during MAVEN cruise. Journal of Geophysical Research: Space Physics, 122, 2089-2105. https://doi.org/10.1002/2016JA023466

McClintock, W. E., Rottman, G. J., \& Woods, T. N. (2005). Solar-stellar irradiance comparison experiment II (Solstice II): Instrument concept and design. Solar Physics, 230, 225-258. https://doi.org/10.1007/s11207-005-7432-x

McClintock, W. E., Schneider, N. M., Holsclaw, G. M., Clarke, J. T., Hoskins, A. C., Stewart, l., et al. (2015). The Imaging Ultraviolet Spectrograph (IUVS) for the MAVEN mission. Space Science Reviews, 195, 75-124. https://doi.org/10.1007/s11214-014-0098-7

McElroy, M. B. (1972). Mars: An evolving atmosphere. Science, 175, 443-445. https://doi.org/10.1126/Science.175.4020.443

McFadden, J. P., Kortmann, O., Curtis, D., Dalton, G., Johnson, G., Abiad, R., et al. (2015). MAVEN SupraThermal and Thermal lon Composition (STATIC) instrument. Space Science Reviews, 195(1-4), 199-256. https://doi.org/10.1007/s11214-015-0175-6

Parkinson, T. D., \& Hunten, D. M. (1972). Spectroscopy and aeronomy of $\mathrm{O}_{2}$ on Mars. Journal Atmospheric Sciences, 29, $1380-1390$. https://doi.org/10.1175/1520-0469(1972)029<1380:SAAOOO>2.0.CO;2 
Rahmati, A., Larson, D. E., Cravens, T. E., Lillis, R. J., Halekas, J. S., McFadden, J. P., et al. (2017). Maven measured oxygen and hydrogen pickup ions: Probing the Martian exosphere and neutral escape. Journal of Geophysical Research: Space Physics, 122, $3689-3706$. https://doi.org/10.1002/2016JA023371

Riley, A. (2017). Stis instrument handbook, version 16.0 (Tech Rep.) Baltimore: STSCl.

Romanelli, N., Mazelle, C., Chaufray, J. Y., Meziane, K., Shan, L., Ruhunusiri, S., et al. (2016). Proton cyclotron waves occurrence rate upstream from Mars observed by MAVEN: Associated variability of the Martian upper atmosphere. Journal of Geophysical Research: Space Physics, 121, 11,113-11,128. https://doi.org/10.1002/2016JA023270

Snow, M., Reberac, A., Quémerais, E., Clarke, J., McClintock, W. E., \& Woods, T. N. (2013). A new catalog of ultraviolet stellar spectra for calibration. Cross-Calibration of Far UV Spectra of Solar System Objects and the Heliosphere, 13, 191-226. https://doi.org/10.1007/978-1-4614-6384-9_7

Terada, K., Terada, N., Shinagawa, H., Fujiwara, H., Kasaba, Y., Seki, K., et al. (2016). A full-particle Martian upper thermosphere-exosphere model using the DSMC method. Journal of Geophysical Research: Planets, 121, 1429-1444. https://doi.org/10.1002/2015je004961

Thiemann, E. M. B., Eparvier, F. G., Bougher, S. W., Dominique, M., Andersson, L., Girazian, Z., et al. (2018). Mars thermospheric variability revealed by MAVEN EUVM solar occultations: Structure at aphelion and perihelion, and response to EUV forcing. ArXiv e-prints.

Villanueva, G. L., Mumma, M. J., Novak, R. E., Kaufl, H. U., Hartogh, P., Encrenaz, T., et al. (2015). Strong water isotopic anomalies in the Martian atmosphere: Probing current and ancient reservoirs. Science, 348(6231), 218-221. https://doi.org/10.1126/science.aaa3630

Woods, T. N. (2005). Solar EUV experiment (SEE): Mission overview and first results. Journal Geophysical Research, 110, A01312. https://doi.org/10.1029/2004JA010765

Yamauchi, M., Hara, T., Lundin, R., Dubinin, E., Fedorov, A., Sauvaud, J. A., et al. (2015). Seasonal variation of Martian pick-up ions: Evidence of breathing exosphere. Planetary and Space Science, 119, 54-61. https://doi.org/10.1016/j.pss.2015.09.013

Yung, Y. L., Wen, J. S., Moses, J. I., Landry, B. M., Allen, M., \& Hsu, K. J. (1989). Hydrogen and deuterium loss from the terrestrial atmosphere: A quantitative assessment of nonthermal escape fluxes. Journal of Geophysical Research, 94(D12), 14,971-14,989. https://doi.org/10.1029/JD094id12p14971

Yung, Y. L., Wen, J. S., Pinto, J. P., Pierce, K. K., \& Allen, M. (1988). HDO in the Martian atmosphere-Implications for the abundance of crustal water. Icarus, 76, 146-159. https://doi,org/10.1016/0019-1035(88)90147-9 